\title{
PALEO
}

Revue d'archéologie préhistorique

\section{Un campement épipaléolithique de chasseurs dans l'ouest de la France}

Le site 1 du Camp d'Auvours à Saint-Mars-la-Brière (Sarthe, France)

An epipalaeolithic camp of hunters in the West of France. The site 1 of the Camp

of Auvours to Saint-Mars-la-Brière (Sarthe, France)

\section{Michel Allard}

\section{OpenEdition}

\section{Journals}

Édition électronique

URL : http://journals.openedition.org/paleo/2516

DOI : $10.4000 /$ paleo.2516

ISSN : 2101-0420

Éditeur

SAMRA

Édition imprimée

Date de publication : 15 décembre 2013

Pagination : 21-46

ISSN : 1145-3370

Référence électronique

Michel Allard, « Un campement épipaléolithique de chasseurs dans l'ouest de la France », PALEO [En

ligne], 24 | 2013, mis en ligne le 17 avril 2014, consulté le 07 juillet 2020. URL : http://

journals.openedition.org/paleo/2516 ; DOI : https://doi.org/10.4000/paleo.2516

Ce document a été généré automatiquement le 7 juillet 2020.

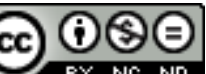

PALEO est mis à disposition selon les termes de la licence Creative Commons Attribution - Pas d'Utilisation Commerciale - Pas de Modification 4.0 International. 


\title{
Un campement épipaléolithique de chasseurs dans l'ouest de la France
}

\author{
Le site 1 du Camp d'Auvours à Saint-Mars-la-Brière (Sarthe, France) \\ An epipalaeolithic camp of hunters in the West of France. The site 1 of the Camp \\ of Auvours to Saint-Mars-la-Brière (Sarthe, France)
}

\section{Michel Allard}

1 La station préhistorique de plein air du Camp d'Auvours, à Saint-Mars-la-Brière (Sarthe), a donné lieu sur son site $\mathrm{n}^{\circ} 1$ à des fouilles dont nous avons eu la responsabilité de 1970 à 1979. A partir de 1983, le changement d'affectation de la DRAC des Pays de la Loire vers la DRAC de Midi-Pyrénées mit fin à cette fouille et nous éloigna de ces recherches.

2 En 1993, la fouille a été détruite accidentellement par les militaires du camp à l'occasion d'un aménagement de la piste qui longe le site.

3 Il nous paraît nécessaire de témoigner de ce que nous avons appris du site 1 d'Auvours, non seulement de son abondant mobilier conservé au musée du Mans mais aussi des observations réalisées lors de la fouille. Même si ces investigations sont restées en deçà de ce qui eut été possible dans le cas d'une fouille conduite à son terme telle que nous l'avions souhaitée, elles constituent néanmoins à travers la présente publication, un témoignage sur les conditions d'étude, les observations, les méthodes mises en œuvre pour optimiser des résultats même incomplets.

4 Il est vrai que, depuis cette époque, la recherche archéologique a beaucoup progressé, en particulier grâce aux développements de l'archéologie de sauvetage et de l'informatique. En tout état de cause, autant l'étude hors sol du mobilier archéologique peut être revue en permanence, autant la lecture du terrain ne peut être validée que lors de la fouille. En l'occurrence, le site 1 d'Auvours atteint par sa destruction prématurée ne peut faire l'objet d'aucune vérification sur le terrain. C'est pourquoi nous tenons à partager ici l'essentiel de ce qu'il nous a appris. 
5 À ce jour, nous avons signé deux publications préliminaires sur le gisement $1 \mathrm{~d}$ 'Auvours (Allard 1982) (Allard et Guyot 1972). Cependant, dans la revue Gallia-Préhistoire, J. L'Helgouach $(1971,1973,1975,1977,1979)$ a publié des rubriques sur le site que nous avions rédigées à sa demande.

\section{1 - Contexte environnemental}

\section{1 - Localisation}

Le camp militaire d'Auvours débute à une quinzaine de kilomètres à l'est du Mans, au nord de la route de Blois près de son intersection avec la R.N.23 (fig. 1-a). Occupant un territoire de forme trapézoïdale large de $400 \mathrm{~m}$ environ à l'ouest, il s'allonge régulièrement vers l'est sur $8 \mathrm{~km}$ jusqu'à la Butte d'Ardenay où sa largeur atteint 1400 $\mathrm{m}$. L'administration militaire est cantonnée à l'extrémité occidentale de cette étendue dont le reste est occupé par un vaste champ de tir. Aux deux tiers de sa longueur, le camp est traversé obliquement du sud-est vers le nord-est par le ruisseau du Narais. La partie concernée par notre étude se situe en rive droite de ce ruisseau près de SaintEtienne-du-Narais, en limite interne du camp séparé de la route de Blois par une lande boisée large d'une soixantaine de mètres appartenant à des particuliers.

Figure 1a - Localisation géographique du gisement du Camp d'Auvours d'après la carte de Bouloire $1 / 50000$.

Figure1b - Localisation cadastrale des sites 1 à 5 .
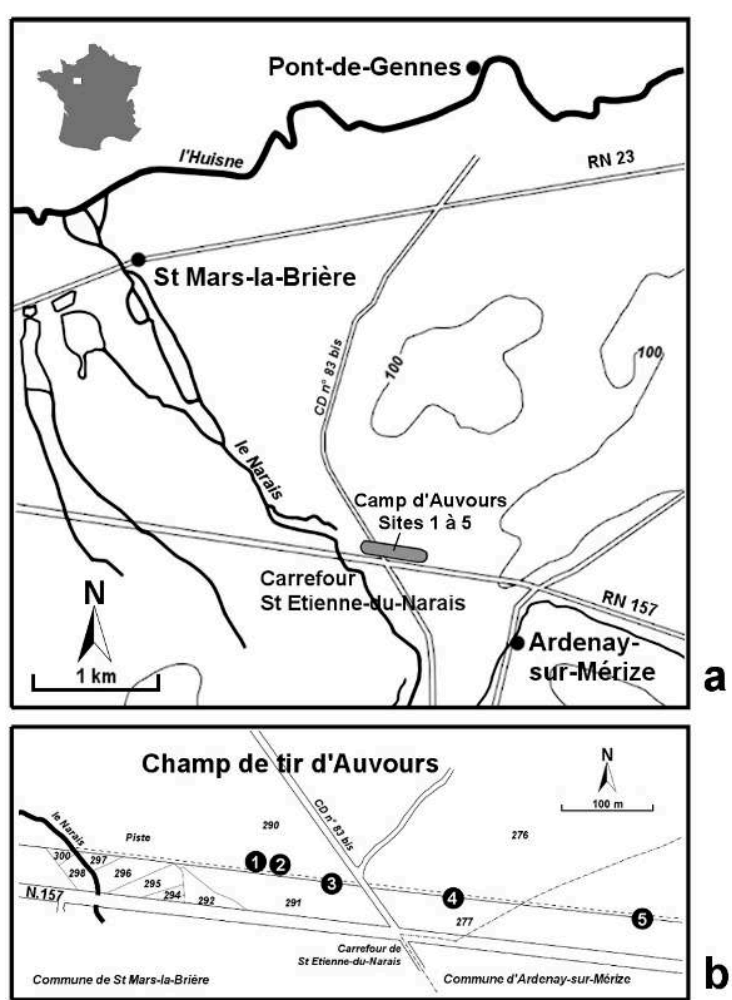


\section{2 - Historique}

7 La station préhistorique du Camp d'Auvours a été découverte en 1969 par R. Guyot, correspondant des Antiquités Préhistoriques, lors d'une prospection réalisée en limite sud du champ de tir entre le ruisseau du Narais et la butte d'Ardenay (fig. 1b, photo 1). À cet endroit, le long de la piste bordant le camp, une bande de terre d'une quinzaine de mètres de largeur avait été décapée de sa couche végétale. Celle-ci, repoussée sur l'autre bord, y formait une butte continue de 1 à $2 \mathrm{~m}$ d'élévation. Le terrain sableux ainsi mis à nu était destiné à servir de pare-feu en cas d'incendie. La découverte sur le sable de nombreux silex taillés incita $\mathrm{R}$. Guyot à $\mathrm{y}$ effectuer une prospection systématique de surface. Il remarqua ainsi cinq concentrations de silex sur une distance d'environ $600 \mathrm{~m}$. Le matériel lithique recueilli constitué de grattoirs, de burins et surtout de nombreuses lamelles à dos, fut dès lors déclaré comme probablement Magdalénien.

8 À la suite de ce signalement à la Direction des Antiquités Préhistoriques, et en accord avec R. Guyot, nous avons effectué plusieurs prospections sur le site. Cela permit de recueillir, sur la bande pare-feu ainsi que dans l'humus du talus latéral, un matériel lithique abondant et de localiser les sites. Ceux-ci dont quatre se rapportent au territoire de Saint-Mars-la-Brière et le cinquième à la commune d'Ardenay-sur-Mérize ont été numérotés d'ouest en est de 1 à 5 . Les trois premiers situés à l'ouest du chemin départemental $n^{\circ} 83$ bis semblent se rapporter à une même culture. En revanche les sites 4 et 5 , moins riches et plus diffus, paraissent correspondre à d'autres niveaux culturels difficiles à préciser. L'enfouissement de ces sites est relativement faible puisqu'un simple décapage du sol végétal a suffi à les faire apparaître. Les sites $n^{\circ} 3,4$ et 5 ont aussi livré du mobilier dans les fossés bordant la piste qui les traverse. Au nord du pare-feu, dans la lande à Éricacées parsemée de bouleaux, des découvertes isolées de mobilier lithique tendent à indiquer la présence d'autres gisements à l'intérieur du champ de tir.

Photo 1 - Vue aérienne du site 1 (cliché J. Rioufreyt, 1972).

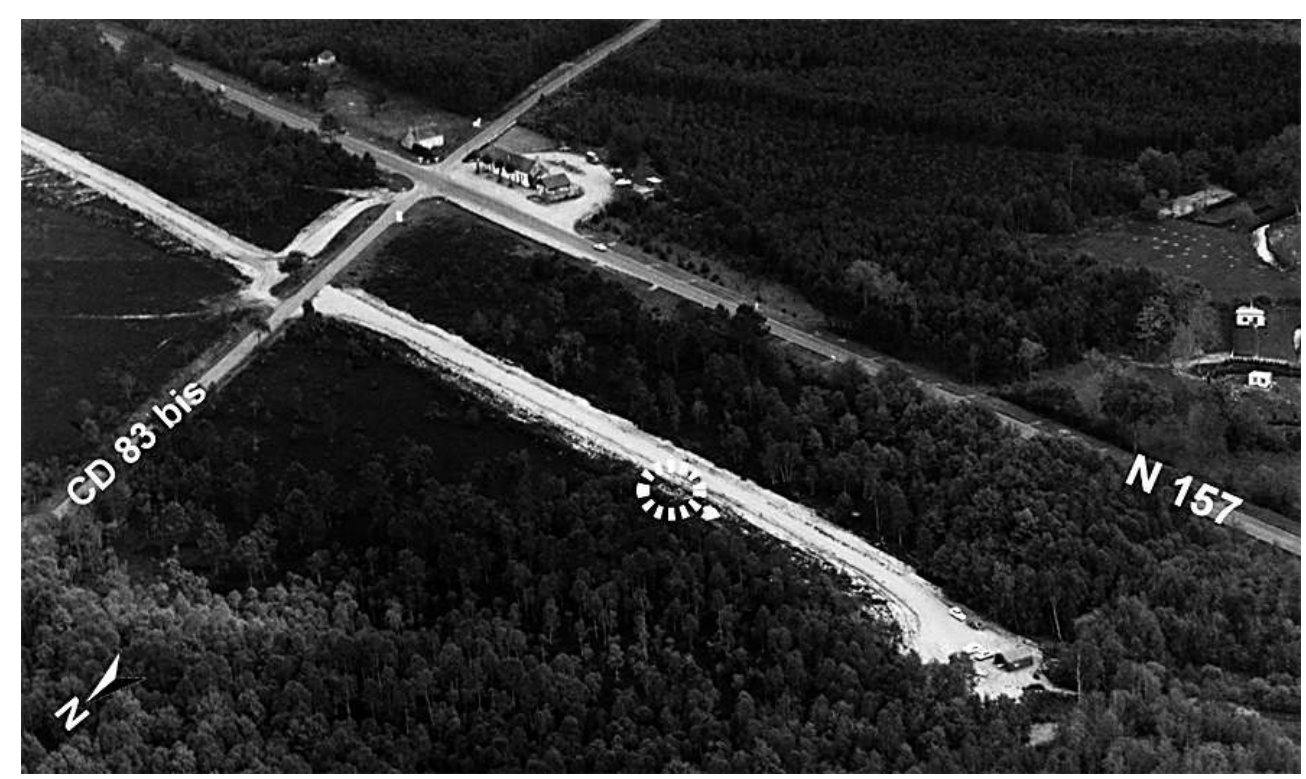

PALEO, 24 | 2013 
Figure 2 - Localisation géologique du site 1, d'après la carte géologique de Bouloire 1/50 000.

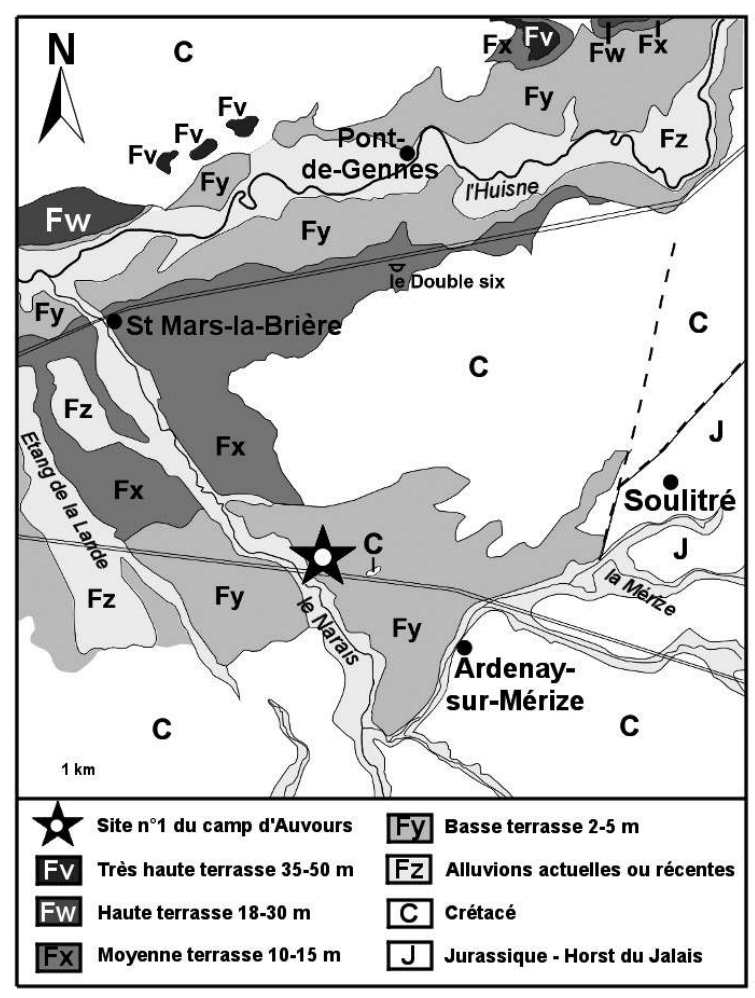

\section{3 - Contexte géologique}

9 Selon la carte géologique (Bouloire 1/50 000) (fig. 2) et les études de Guy Mary (Mary 1964), géologue à l'Université du Maine et spécialiste des environs du Mans, avec lequel nous nous étions entretenu à propos de la fouille du site 1, l'environnement du gisement est formé d'alluvions anciennes déposées à des altitudes variées par rapport au lit majeur du Narais environné de marécages près de sa confluence avec l'Huisne. Ces alluvions disposées en terrasses emboîtées, mais en partie démantelées par l'érosion, n'ont conservé ici que leurs niveaux les plus récents (basse terrasse de 2-5 m et moyenne terrasse de 8-15 m). En revanche, sur la rive droite de l'Huisne subsistent encore quelques vestiges des hautes $(18-30 \mathrm{~m})$ et très hautes $(35-50 \mathrm{~m})$ terrasses. Les redans des hautes terrasses étagées censées marquer la rive gauche de l'Huisne au début du Quaternaire ont donc progressivement été détruits par l'érosion. Ainsi, au niveau du Camp d'Auvours, le relief était sans doute assez proche de son état actuel lorsque au Tardiglaciaire les sites préhistoriques 1 à 4 ont été installés sur la basse terrasse en rive droite du Narais, et pour le site 5 sur le versant d'une petite butte témoin cénomanienne.

La basse terrasse d'Auvours est constituée de sables et de graviers auxquels se mêlent quelques débris d'un calcaire jurassique affleurant six kilomètres à l'est dans le horst du Jalais. La notice de la carte géologique au 1/50.000 de Bouloire (Obert 1988 - p. 24) indique que la terrasse " [contient] des blocs de grès charriés par des radeaux de glace (Saint-Mars-la-Brière) ». À l'emplacement du site 1, des blocs ainsi transportés y constituent un pavement naturel. Selon la carte, cette formation dans laquelle il «a été retrouvé des restes de l'industrie moustérienne serait d'âge wurmien ». Nous ignorons 
pour l'instant l'étendue du pavement qui, dans la zone étudiée, ne concerne que le site 1. En l'occurrence, la fouille a montré que lorsque les préhistoriques se sont installés, le pavement avait déjà été affecté au Paléolithique supérieur par des marques de gélifraction et d'une forte corrasion. Après son abandon, l'ensemble du site a été recouvert par une couche sableuse dont le géologue G. Mary nous a confirmé récemment, en 2013, la constitution d'éléments ruisselés et soufflés.

Il est regrettable que nous n'ayons pas pu consacrer davantage de temps à rechercher l'origine du silex gris utilisé par les occupants préhistoriques du Camp d'Auvours. Les seuls blocs bruts observés étaient situés dans des terrains argileux à proximité du gisement, près de Saint-Denis-du-Tertre à $1500 \mathrm{~m}$ vers le nord, mais les volumes rencontrés ont paru trop petits pour être exploitables. En revanche, la meulière également utilisée sur le site a pu être prélevée sans difficulté dans son proche environnement, en particulier au sud dans le bois de Loudon où des militaires du camp nous en ont fait découvrir de gros blocs résiduels.

En fonction de ces données géologiques et compte tenu du fait que nous n'avons découvert aucun matériel brut taillable sur le site 1, nous ne pouvons suivre l'option avancée par N. Naudinot (Naudinot 2008 - p. 259) concernant l'apport soliflué de matière première lithique taillable.

\section{4 - L'environnement préhistorique : les autres sites découverts}

\subsection{1 - Le site 2}

Le site 2 rencontré dans la bande pare-feu, était localisé entre $140 \mathrm{~m}$ et $145 \mathrm{~m}$ à l'ouest de la borne des $800 \mathrm{~m}$ du CD 83 bis. Également détruit, il a semblé correspondre à la même culture que le site 1 . Cependant, il n'y a été remarqué aucun vestige de pavement. Les prospections superficielles ont livré 216 silex dont deux nuclei, deux pointes à dos proximales et deux fragments distaux de lamelles ou pointes à dos proximales ainsi que 16 plaquettes ocrées rouges. Il constituait peut-être un site plus petit ou plus profondément enfoui que le site 1 .

\subsection{2 - Le site 3}

14 Ce site était coupé par la piste militaire à une distance comprise entre $60 \mathrm{~m}$ et $70 \mathrm{~m}$ à l'ouest de la borne des $800 \mathrm{~m}$. En 1979, un ravivage des fossés bordant la piste occasionna une intervention de sauvetage qui révéla une installation préhistorique marquée par un épais niveau de combustion. Les récoltes y ont livré 847 silex dont cinq nuclei prismatiques et une seule plaquette ocrée. L'outillage comprend en particulier neuf grattoirs, quatre burins, neuf armatures et trois lames tronquées. Très comparable au site 1 par son mobilier lithique, le site 3 s'en différencie cependant par l'absence de blocs.

\subsection{3 - Le site 4}

15 Il s'agit d'une installation humaine traversée par la piste militaire entre $120 \mathrm{~m}$ et $140 \mathrm{~m}$ à l'est de la borne des $800 \mathrm{~m}$ déjà citée. Le mobilier découvert est constitué par 83 silex, dont neuf témoins laminaires et 20 lamellaires, un burin sur cassure et une pointe à dos. 


\subsection{4 - Le site 5} versant d'une petite colline, entre $400 \mathrm{~m}$ et $450 \mathrm{~m}$ à l'est de la borne des $800 \mathrm{~m}$. Elle a livré 265 éclats et débris, 35 lamelles brutes, 53 lames brutes, et cinq nuclei à lames. L'outillage comporte une dizaine de pièces dont un petit grattoir en éventail, un perçoir, un burin sur cassure, deux armatures et un triangle scalène. Le site a aussi fourni un rognon de silex pourvu d'une large géode ouverte et d'une sorte de manche (photo 2) évoquant grossièrement les deux parties du brûloir de Lascaux (Glory 1961). Ce rognon partiellement craquelé et rougi par le feu porte, sur le bord de sa géode, une trace très nette de brûlure pouvant correspondre à l'emplacement d'une mèche. Il semble donc qu'il ait été sélectionné pour sa forme naturelle par les préhistoriques, en vue d'une utilisation comme lampe à graisse dont il présente des stigmates assez caractéristiques.

Photo 2 - Site 5. Nodule de silex avec géode (cliché M. Allard).

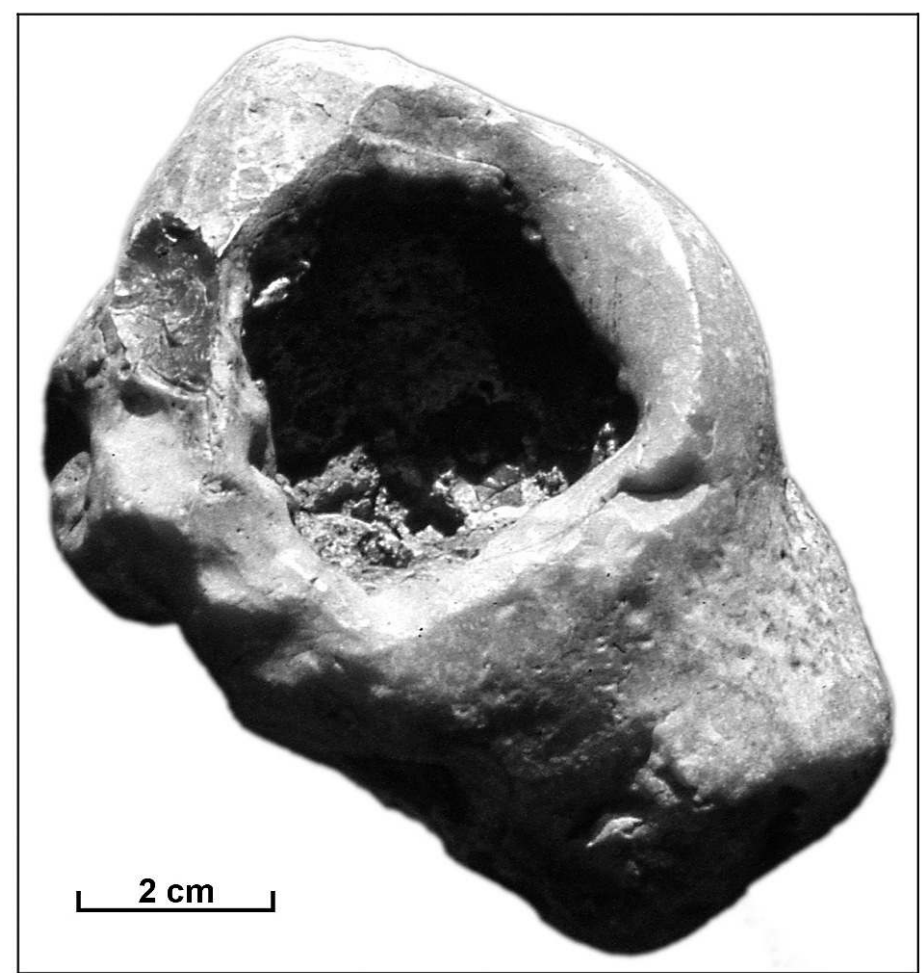

\section{2 - Technique de recherche et problématique appliquées au site 1}

\section{1 - Travaux préliminaires : tamisages et sondages}

\subsection{1 - Tamisages}

17 C'est en 1970 que démarrèrent les premiers travaux sur le site 1, le plus riche en matériel archéologique dans l'emprise du pare-feu (fig. 3). Ayant d'abord supposé le 
terrain remanié, nous y avions entrepris un tamisage sur une surface de 4 mètres carrés. Très vite, il est apparu qu'à partir de 2 ou $3 \mathrm{~cm}$ de profondeur le podzol contenant la couche archéologique était intact. Dès lors, le tamisage fut arrêté dans ce secteur et reporté sur les déblais constituant le talus tout proche. Ceux-ci riches en humus nécessitèrent un recours au tamisage par trempage pour en extraire le mobilier préhistorique. La mise à disposition par les militaires d'une citerne d'eau facilita cette opération qui rapporta plus de 2600 silex, dont 77 outils, et permit une nouvelle attribution du site au Paléolithique terminal.

\subsection{2 - Sondages}

18 Avant d'entreprendre une fouille il convenait d'évaluer plus précisément le potentiel archéologique du site 1 et de définir son emplacement par une série de sondages géographiquement orientés (fig. 3). Six sondages ( $\mathrm{S} 1$ à $\mathrm{S6}$ ) furent donc réalisés. Leur superficie d'un mètre carré au départ ( $\mathrm{S} 1, \mathrm{~S} 2, \mathrm{~S} 3)$ fut réduite de moitié par la suite ( $\mathrm{S} 4$, S5, S6).

Figure 3 - Localisation des travaux.

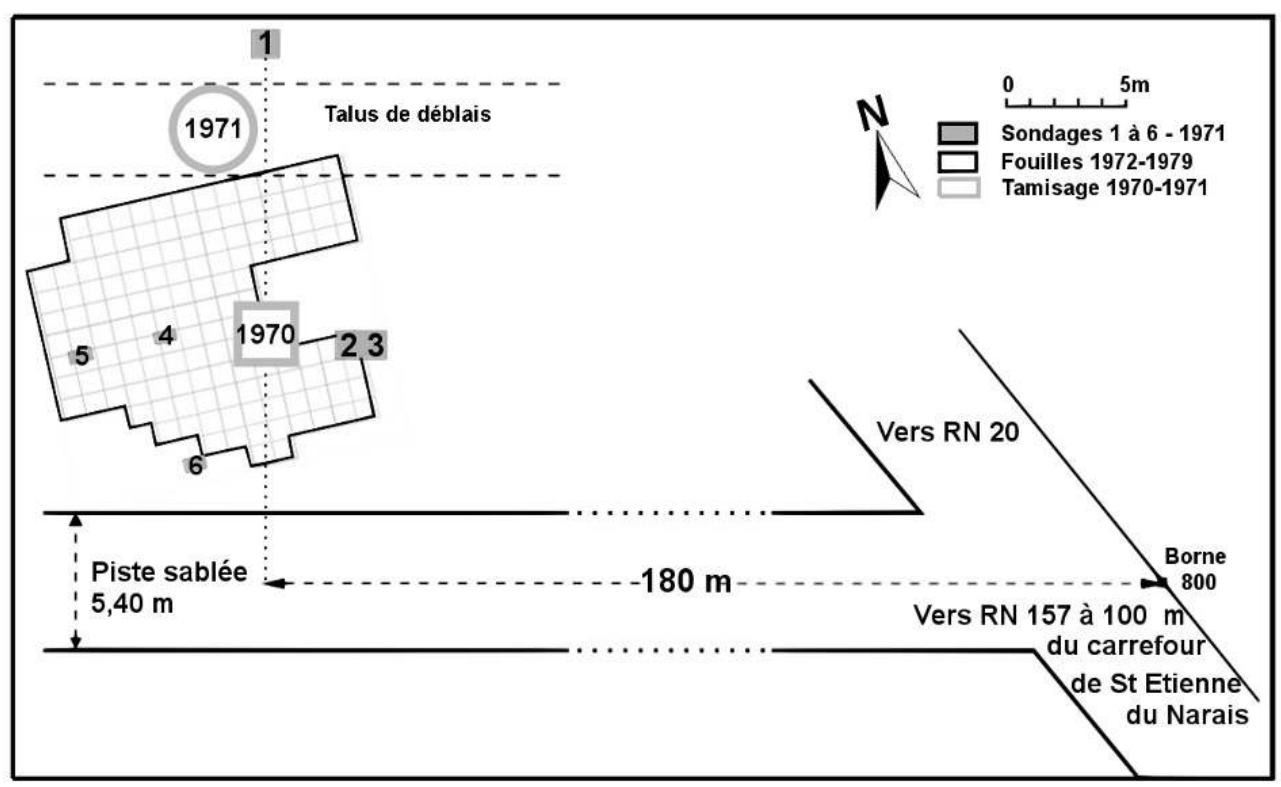

Le sondage S1 avait pour objet l'établissement d'une coupe complète du sol. Il a donc été implanté dans la lande intacte, à $20 \mathrm{~m}$ au nord de l'axe de la piste. Sa richesse matérielle (385 silex) a montré son rattachement probable au site 1. Sa stratigraphie rapportée à l'époque aux travaux de G. Aubert. et J. Boulaine (1967) sur la pédologie et plus récemment à ceux de P. Duchaufour (2001) et de D. Baize (2000 et 2005) a livré les résultats suivants :

- 0-2 cm : humus brut brun-noir avec quelques débris végétaux identifiables (horizon $\mathrm{OF})$.

- 2-20 cm: couche brun-foncé finement sableuse, très riche en matière organique (humus grossier), contenant quelques éléments gréseux blanchis en surface (horizon $\mathrm{OH})$.

- 20-25 cm : sable brunâtre s'éclaircissant vers la base (horizon A). 
- 25-45 cm: couche gris-clair bien développée, sablo-limoneuse et caillouteuse, essentiellement quartzeuse. Il s'agit de l'horizon E à structure cendreuse du podzol.

- 45-55 cm: couche noire très compacte, organique, limono-caillouteuse. Elle caractérise l'horizon BPh (horizon spodique).

$$
\begin{aligned}
& \text { 7-B) } \\
& \text { sud. }
\end{aligned}
$$

\section{2 - Technique de fouille}

Un quadrillage du site 1 orienté sur le nord magnétique de façon à y intégrer les précédents sondages S4, S5, S6 a été établi en 1971. Il a été divisé en sections de 5 mètres de côté désignées par un chiffre romain et subdivisées en mètres carrés nommés chacun par une lettre. Le niveau zéro a été défini par rapport à un gros bloc solidement ancré dans l'angle nord-ouest du sondage S5 (carré 7-B) et dont le sommet peint en rouge correspondait à la cote $-45 \mathrm{~cm}$. Les coordonnées verticales relatives à ce point ont été relevées à l'aide de niveaux à eau répartis de façon à couvrir les 141 mètres carrés fouillés. Cependant, pour faciliter la lecture de la présente publication, les repères de carroyage utilisés sur le terrain ont été transcrits ici en un système plus simple dans lequel les unités métriques sont représentées par des lettres sur l'axe des abscisses et par des chiffres sur celui des ordonnées. Le travail de fouille a été effectué sous abri sur plancher mobile (photo 3 ). 
Photo 3 - Un aspect de la fouille en 1977 (cliché M. Allard)

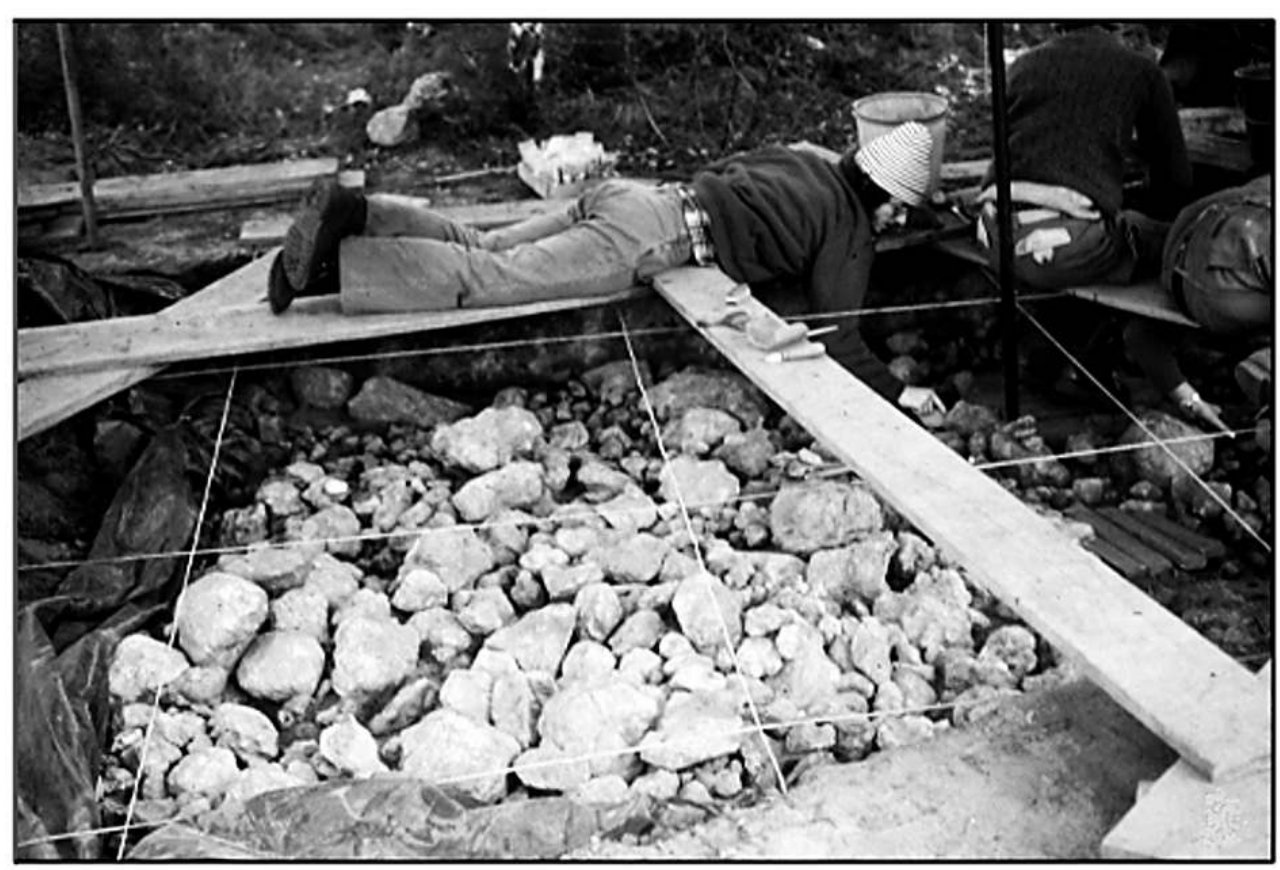

\subsection{1 - Technique appliquée au mobilier lithique}

Les premières années, le mobilier lithique prélevé lors de la fouille a été numéroté individuellement et repéré suivant les trois axes XYZ. Son diagramme de répartition verticale (fig. 4) situe le niveau archéologique entre $40 \mathrm{~cm}$ et $50 \mathrm{~cm}$ du zéro général sauf au niveau des carrés $7 \mathrm{G}$ et $7 \mathrm{H}$ (foyer F1) où il est un peu plus profond. La dispersion verticale du matériel archéologique serait attribuable aux animaux fouisseurs et aux racines. Par ailleurs, un tamisage à sec au tamis de $1,7 \mathrm{~mm}$ des sédiments de chaque carré suivi du trempage dans une bassine d'eau des résidus de ce tamisage facilita la récupération optimale du mobilier.

\subsection{2 - Technique appliquée aux blocs gréseux}

La photographie verticale de chaque carré fouillé a permis la réalisation d'un assemblage photographique de la totalité des blocs gréseux apparents dans la zone fouillée (photo 4). Au début, ceux-ci ont été relevés et numérotés de la même façon que le reste du mobilier. Ainsi, dès la première année, le démontage des blocs du foyer F1 a montré l'aménagement et le caractère anthropique de leur accumulation. Ce constat fut déterminant pour la suite de la fouille. Dès lors, les blocs ont été laissés en place pour en permettre une vision d'ensemble et une étude ultérieure. 
Figure 4 - Graphique de répartition du mobilier archéologique en fonction de la profondeur dans les carrés 7-E, 7-F, 7-G, 7-H, 7I.

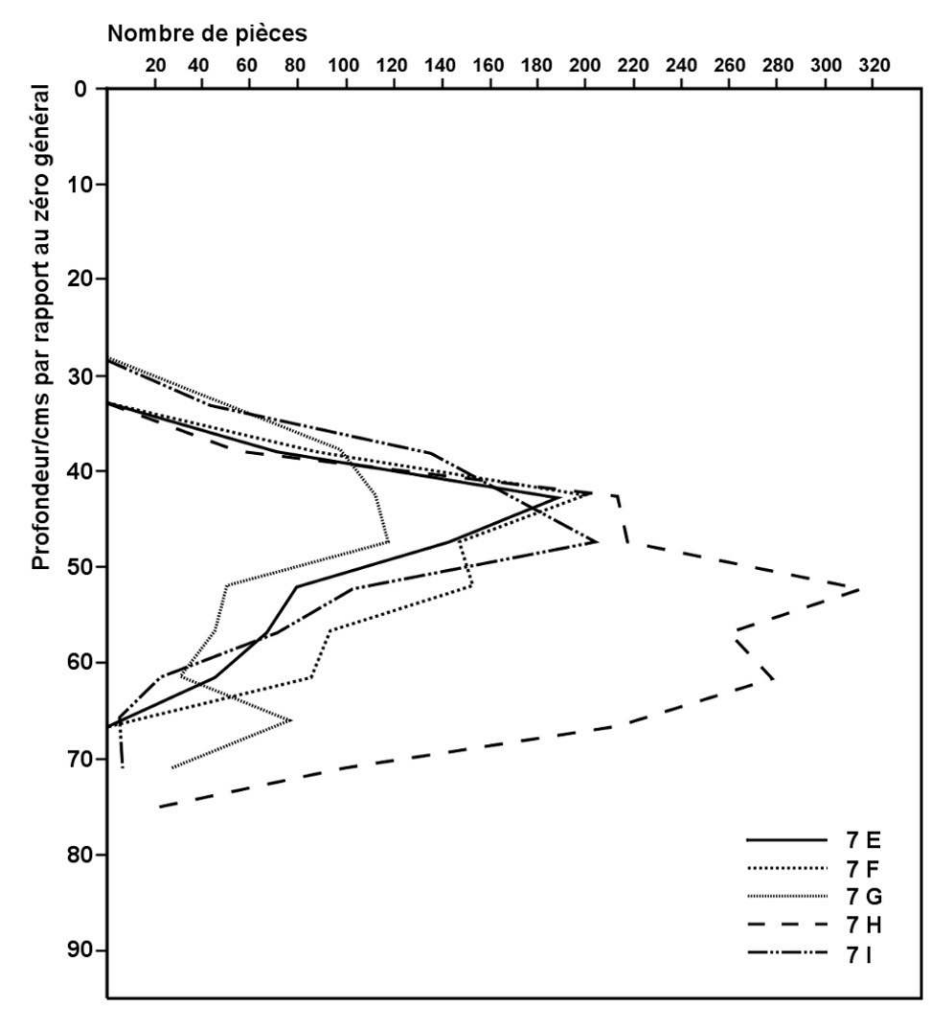

\subsection{3 - Problématique de recherche}

La première année de fouille ayant livré un mobilier lithique abondant et révélé la présence du foyer F1, élément structuré très marqué, nous avons choisi d'orienter la recherche dans deux directions : d'une part, la récupération systématique du mobilier lithique pour en obtenir des informations statistiques d'intérêt culturel et chronologique; d'autre part, l'adaptation de la méthode de travail à l'étude d'une organisation éventuellement plus vaste du site. L'objet final de notre problématique était ainsi une approche palethnologique de ce site à travers toutes ses données, tant celles de sa fouille que des études paléo-environnementales.

\section{3 - Présentation de la série lithique du site 1}

\section{1 - Historique de l'étude}

L'exposé suivant est le résumé d'une analyse du matériel lithique retouché effectuée entre l'arrêt de la fouille et la fin de l'année 1982. Déjà, une importante recherche de raccords nous avait permis la reconstitution totale ou partielle de nombreuses armatures qui, sans cela, auraient été difficilement imaginables. Cette analyse intégrant la description et les mesures précises de chaque témoin retouché a été complétée par des décomptes statistiques et des plans de répartition relatifs aux diverses catégories d'objets. Pour des raisons de place, seule une infime partie du millier de pièces dessinées au cours des années 1979 et 1980 est présentée dans cet article. 
Comme l'étude technologique des industries lithiques du site 1 a été actualisée (Naudinot 2008), notre exposé sur le mobilier retouché concerne essentiellement des observations et des statistiques effectuées autour des années 1980 et qui n'ont pas été traitées dans l'étude technologique citée. Elles se rapportent notamment aux grattoirs et à certaines armatures du gisement.

Photo 4 - Assemblage photographique du site préhistorique d'Auvours (cliché M. Allard).

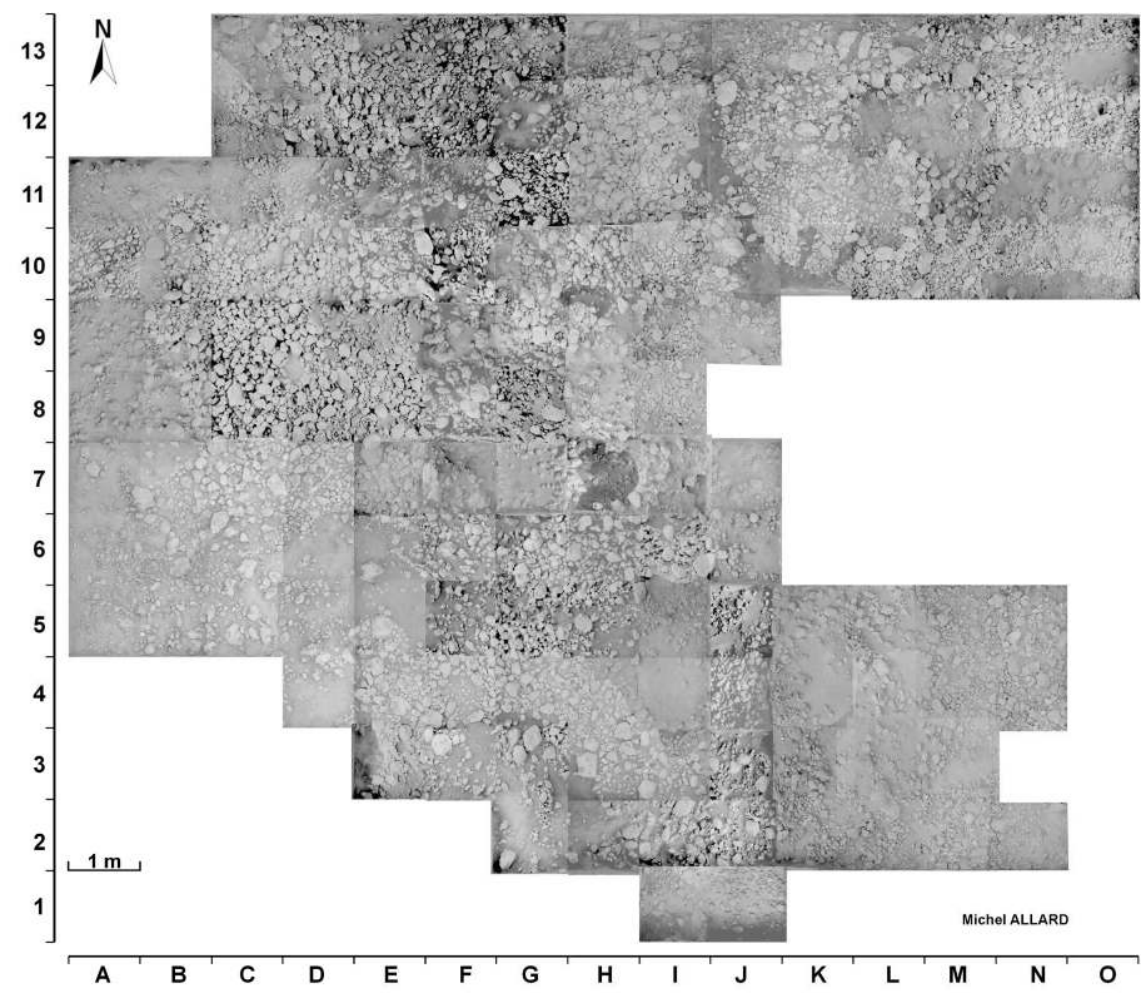


Figure 5 - Répartition chiffrée, dans la fouille, du mobilier lithique débité.

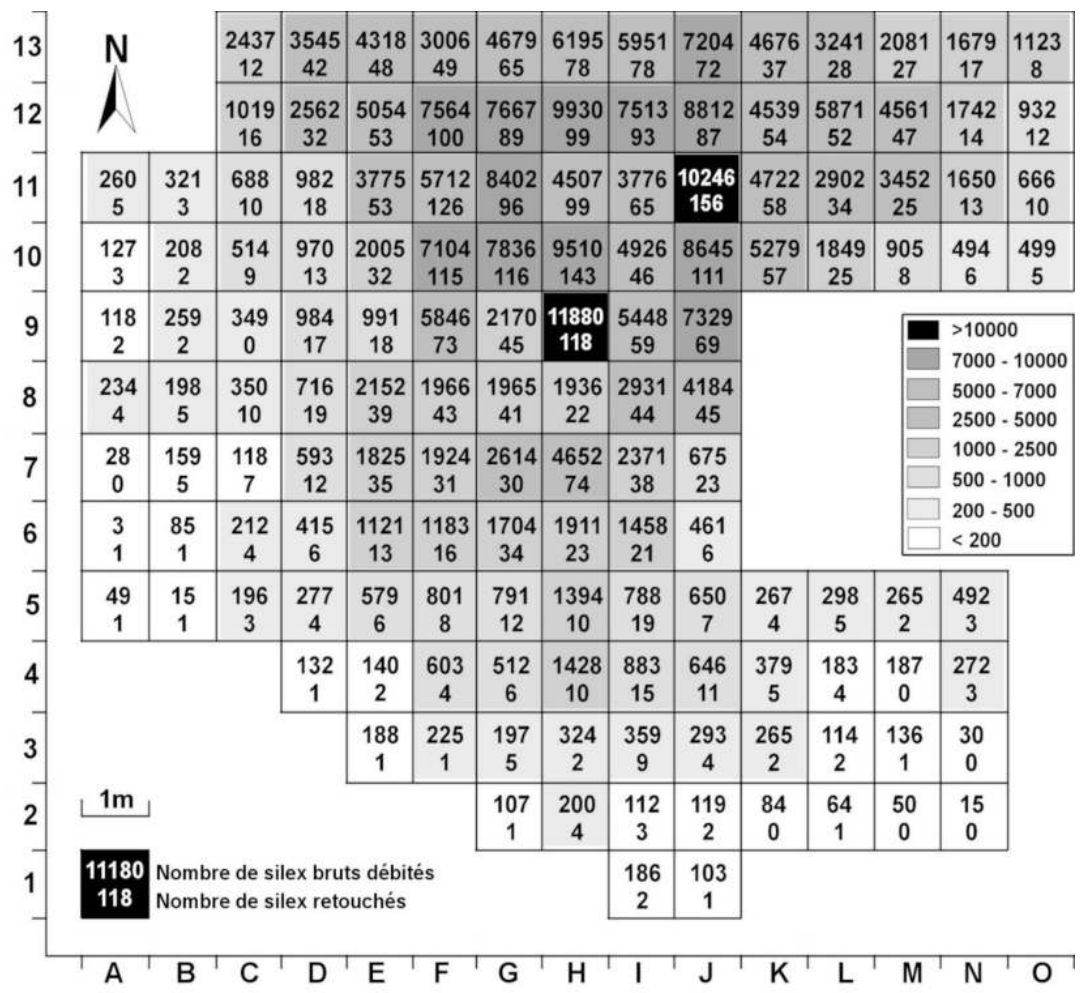

\section{2 - Composition générale de l'assemblage lithique}

31 Le mobilier préhistorique du site 1, exclusivement minéral, compte surtout une quantité considérable de témoins lithiques débités (environ 327000 pièces) représentant, pour la plupart, des produits bruts intégrant une part très importante de micro-éclats et d'esquilles. La concentration de ce mobilier dans la moitié nord où sa densité peut atteindre plus de 10000 silex au m2 (fig. 5) est interrompue par la limite de la zone fouillée. Des matières colorantes et des charbons de bois ont aussi été recueillis. Enfin, le site a livré quelques objets lithiques non taillés : grès rainurés, billes.

\subsection{1 - Les matériaux débités}

32 Le silex constitue le matériau essentiel présentant plusieurs qualités. La variété meulière exploitée à faible distance du site, à partir de petits blocs et de plaquettes, a été très utilisée pour la confection d'outils du fond commun n'exigeant pas de qualités particulières. Par ailleurs, un silex gris moucheté de blanc, matériau d'excellente qualité employé pour la fabrication d'armatures et de grattoirs, est à rechercher hors de l'environnement immédiat du gisement. Quelques fragments de grès quartzite d'origine incertaine ont été trouvés.

\subsection{2 - Les nuclei et les percuteurs}

Il a été décompté 214 nuclei dont 67 présentent des traces de chauffage souvent marquées par un craquèlement interne ou par des cupules thermiques. Leurs volumes modestes présentent des longueurs comprises entre $93 \mathrm{~mm}$ et $27 \mathrm{~mm}$ avec une 
moyenne de $47,5 \mathrm{~mm}$. Il s'agit surtout de nuclei prismatiques à un $(25 \%)$ ou deux (35\%) plans de frappe. Leur forme, bien que parfois cylindrique (fig. 6a), apparaît le plus souvent comprimée entre une tablette de débitage aplatie et un dos en partie cortical (fig. 6b). D'autres types de nuclei à plans de frappe alternes, croisés ou multiples représentent chacun environ $5 \%$ de l'ensemble. Nous n'avons rencontré que deux nuclei bipyramidaux, deux autres polyédriques à éclats et un seul nucleus conique. Le débitage paraît orienté vers une production lamino-lamellaire. Cependant, il existe aussi une importante production d'éclats dont certains, envahis de cortex, seraient issus de phases de décorticage. Néanmoins, d'autres produits pourvus de restes corticaux semblent, par souci d'économie, avoir été débités sans décorticage préalable dans des volumes de très petite dimension.

Figure 6

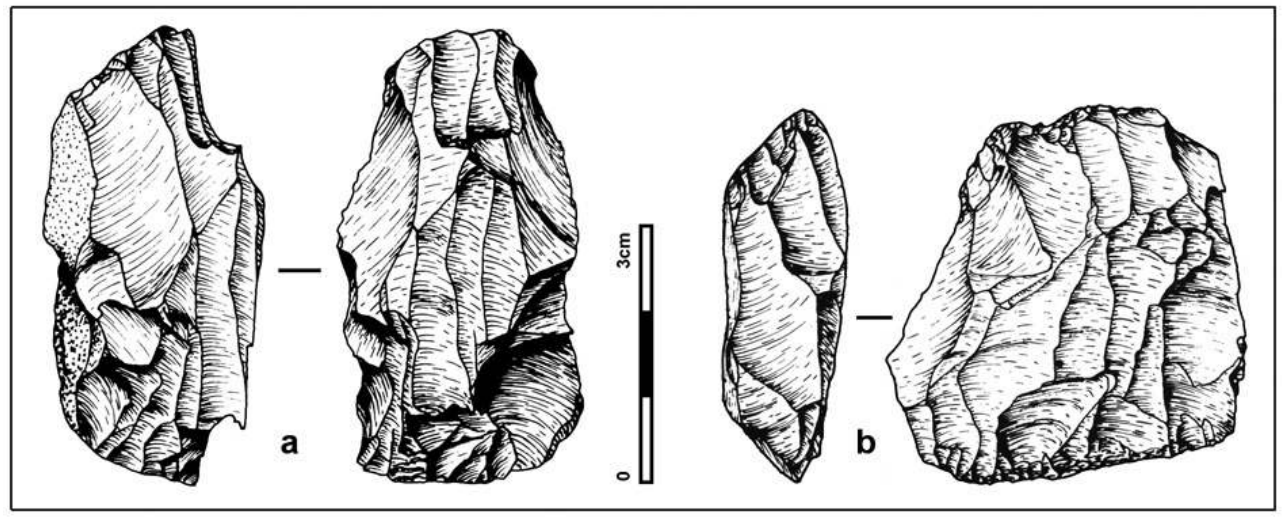

a - Nucleus prismatique à plans de frappe opposés.

b - Nucleus aplati à deux plans de frappe (dessin M. Allard).

Malgré la profusion de mobilier et la présence de nombreux nuclei, le site n'a livré aucun percuteur. Par ailleurs, l'étude de nombreux supports lamino-lamellaires dont les talons ont été systématiquement abrasés indique que ceux-ci n'ont pas été taillés à l'aide de bois animal ou végétal. Cela incline à penser que les percuteurs étaient soit en pierre tendre comme le suggère la proximité des gisements de calcaire, soit qu'ils ont été emportés. Malheureusement, la nature très siliceuse du sol ne nous permet pas de conclure.

\section{3 - Le matériel lithique retouché}

L'inventaire compte près de 4200 témoins lithiques retouchés soit 1,28 \% du mobilier du site 1. Ce décompte a été ramené à 3899 pièces par la réalisation d'assemblages (tabl. 1). En effet, les trois-quarts de ce mobilier, constitué de lamelles avec dos très fragmentaires, ont permis la réalisation d'environ 300 raccords.

Les grattoirs comportant 244 outils simples comptent 265 fronts si l'on décompte ceux des outils doubles et des pièces composites.

De même, les burins représentés normalement par 231 pièces offrent 314 biseaux si l'on considère aussi ceux des pièces multiples et composites.

Ces décomptes soulignent le relatif équilibre entre les burins et les grattoirs avec un rapport grattoirs/burins à peine supérieur à l'unité lorsqu'on le calcule de façon 
classique $(244 / 231=1,05)$ mais plus faible lorsqu'on y intègre les éléments multiples et composites $(274 / 322=0,84)$. Cette proportion de burins imprime un net caractère paléolithique à la série.

Contrairement à la série brute, le mobilier retouché montre une utilisation prépondérante des lames et des lamelles comme supports. En particulier, les armatures ont toutes été exécutées sur lamelles ou, à défaut, sur petites lames rétrécies par la retouche.

Tableau 1 - Décompte du mobilier retouché.

\begin{tabular}{|l|c|c|}
\hline Catégorie & Nombre & $\%$ \\
\hline Grattoir & 244 & 6,25 \\
Burin & 231 & 5,92 \\
Outil composite & 32 & 0,82 \\
Perçoir ou bec & 29 & 0,74 \\
Lame à bord abattu & 3 & 0,07 \\
Lame, retouche continue & 3 & 0,07 \\
Encoche & 39 & 1 \\
Denticulé & 9 & 0,23 \\
Raclette & 23 & 0,58 \\
Lame ou éclat tronqué & 90 & 2,3 \\
Pièce bitronquée & 12 & 0,3 \\
Lamelle bitronquée & 153 & 3,92 \\
Microperçoir & 101 & 2,54 \\
Lamelle à encoche & 41 & 1,05 \\
Triangle & 4 & 0,1 \\
Lamelle à dos et/ou troncature & 2844 & 72,94 \\
Divers & 41 & 1,05 \\
Totaux & 3899 & 99,85 \\
\hline
\end{tabular}

\subsection{1 - Les grattoirs}

Les 244 grattoirs (fig. 7) occupent majoritairement une position distale sur les supports. Le pourcentage des fronts proximaux y atteint cependant $13,2 \%$ (tabl. 2).

Tableau 2 - Étude des grattoirs.

\begin{tabular}{|l|c|c|c|c|c|c|c|c|c|c|c|}
\hline Type de grattoir & Nombre & \multicolumn{3}{|c|}{ Dimensions } & \multicolumn{3}{c|}{ Localisation du front } & \multicolumn{3}{c|}{ Orientation du front } \\
\hline & & L & I & é & Proximal & Distal & Axial & Dextre & Sénestre & Irrésolu \\
\hline G. raccourci & 94 & 27,7 & 23,5 & 7,67 & 9 & 85 & $37(41,5 \%$ & $38(42,6 \%)$ & $14(15,7 \%)$ & 5 \\
G. brisé & 39 & & & & 9 & 30 & 3 & 5 & 1 & 30 \\
G. sur lame & 17 & 42,66 & 20 & 7,5 & 1 & 16 & 7 & 6 & 1 & 3 \\
G. double & 6 (12 fronts) & 32,8 & 25,66 & 9,66 & 6 & 6 & 0 & 2 & 2 & 2 \\
G. sur éclat & 56 & 37,1 & 28,13 & 8,8 & 2 & 54 & 30 & 22 & 2 & 2 \\
G. caréné & 9 & 43 & 21,25 & 0 & 1 & 8 & 0 & 5 & 3 & 1 \\
G. épaulement & 23 & 30,46 & 25,29 & 7,65 & 4 & 19 & & & & \\
\hline Totaux & 244 & & & & 32 & 218 & 77 & 78 & 23 & 43 \\
& & & & & $13,2 \%$ & $89,3 \%$ & $34,84 \%$ & $35,29 \%$ & $10,40 \%$ & $19,45 \%$ \\
\hline
\end{tabular}

41 Quatre grattoirs présentant sous le front une retouche inverse plus ou moins esquilleuse ont pu servir d'herminettes ou de pièces intermédiaires pour le travail de matières dures. 

esaisseur (é). L'objectif de cette étude est de fournir des éléments de marquage permettant d'établir des comparaisons et d'éventuels liens avec d'autres séries.

Figure 7- Grattoirs. Grattoir raccourci : nos 1-18. Grattoir sur lame : nos 19-20. Grattoir double : $n^{\circ} 25$. Grattoir sur éclat : nos 21-24. Grattoir caréné : $n^{\circ} 27$. Grattoir à épaulement : $n^{\circ} 26$ (dessin $M$. Allard).

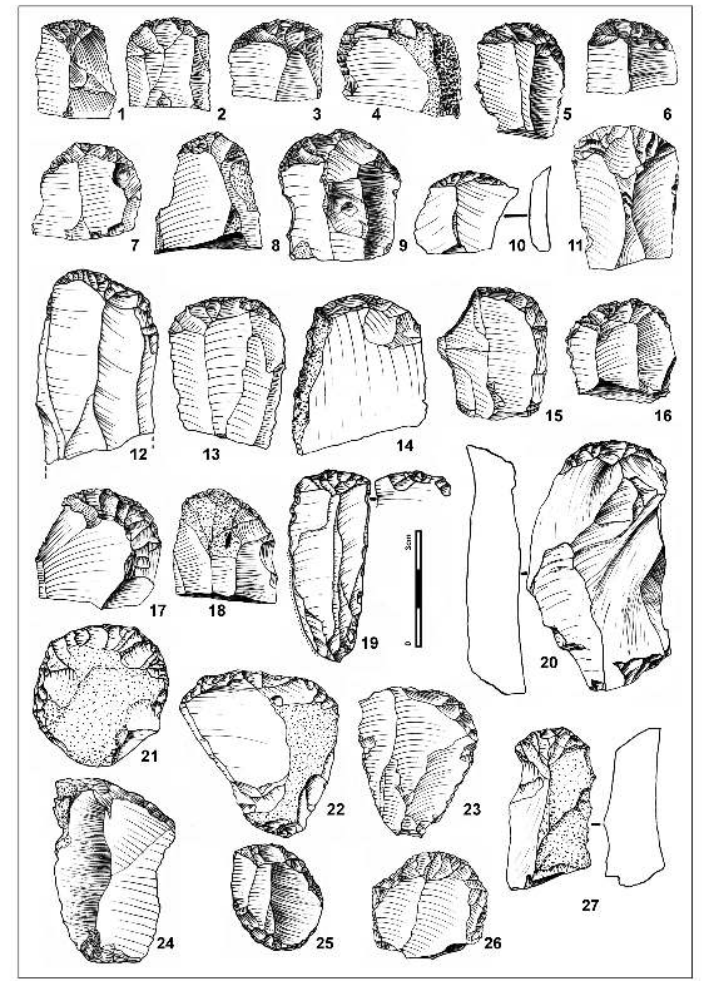

\subsubsection{1 - Grattoirs raccourcis}

Ce sont des grattoirs sur fragments de lames ou d'éclats laminaires dont l'extrémité opposée au front a été fracturée par flexion directe ou inverse et dont la longueur est inférieure au double de la largeur. Ces outils, représentés par 94 pièces, constituent la portion la plus importante, soit 37,75\% du groupe des grattoirs (fig. 7, nos 1-18). C'est pourquoi ils se prêtent le mieux à une étude métrique statistique.

Leurs longueurs, comprises entre 25 et $30 \mathrm{~mm}$, présentent une moyenne de $27,7 \mathrm{~mm}$.

Leurs largeurs se situent entre 20 et $25 \mathrm{~mm}$ avec une moyenne de $23,5 \mathrm{~mm}$.

Leurs épaisseurs de 4 à $13 \mathrm{~mm}$ présentent une moyenne de 7,67 $\mathrm{mm}$ avec toutefois une fréquence maximale pour l'épaisseur de $6 \mathrm{~mm}$.

Il a paru intéressant aussi de mesurer l'inclinaison sagittale du front de chaque grattoir sur sa face plane. Cette inclinaison inscrite entre $35^{\circ}$ et $80^{\circ}$ présente un maximum pour 
les valeurs comprises entre $50^{\circ}$ et $60^{\circ}$. L'ensemble de ces résultats conforte l'homogénéité de la série.

Par ailleurs, dans 75 \% des cas, la fracture opposée au front du grattoir raccourci est directe, c'est-à-dire produite à partir de la face plane vers la face dorsale de l'outil. Elle peut, dans ce cas, présenter une languette ou son négatif, du côté dorsal. De plus, $40 \%$ de ces grattoirs raccourcis par fracture directe présentent un témoin de languette assorti d'esquilles attestant la violence du choc qui a généré la cassure. Ce type de fracture semble difficile à réaliser sur un grattoir tenu à main nue. En revanche, son emmanchement peut permettre de le lancer avec assez de force pour produire un choc fronto-ventral capable de le briser de cette façon (fig. 8). Enfin, une dizaine de ces grattoirs raccourcis associés à une fracture directe portent aussi des retouches ou des encoches latérales ou bilatérales en bord de fracture. Celles-ci peuvent correspondre à des emplacements de ligatures. L'ensemble de ces observations constitue ainsi un faisceau d'arguments en faveur de l'emmanchement de ces grattoirs. Ce ne serait pas le cas pour les grattoirs raccourcis présentant une fracture inverse.

L'absence d'assemblage entre fragments distaux et proximaux de tels outils suggère que leurs parties proximales réutilisées ne sont plus identifiables.

Enfin, trois types de fronts, en relation probable avec un usage particulier, ont été distingués à l'intérieur de cette catégorie de grattoirs : les fronts axiaux (41,5\%) dont l'axe correspond à celui du support, ceux déjetés à droite $(42,6 \%)$ et ceux déjetés à gauche $(15,7 \%)$. Le type déjeté à droite est dominant. Si l'on y associe les fronts déjetés à gauche, le taux de latéralisation de ces grattoirs atteint la proportion remarquable de $58,3 \%$.

51 Le cortex présent sur plus du quart de ces grattoirs peut traduire le fait que leurs supports correspondent au décorticage de rognons de silex de petite dimension.

Figure 8 - Mode possible de raccourcissement par fracture d'un grattoir (dessin M. Allard)

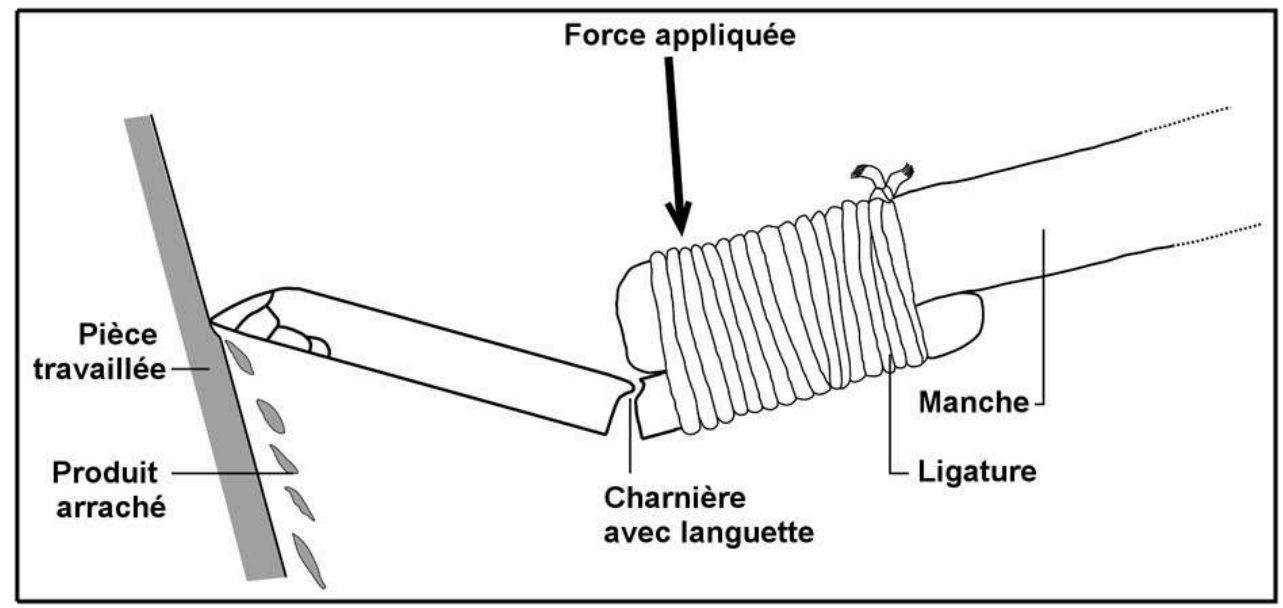

\subsubsection{2 - Autres types de grattoirs}

Des études comparables ont été effectuées sur les autres grattoirs. Leurs résultats se trouvent consignés dans le tableau 2. 


\subsection{2 - Les burins}

La suite de l'outillage (fig. 9) est marquée par le groupe des burins constitué par 232 outils dont la répartition est indiquée sur la tableau 3.

Tableau 3 - Décompte des burins.

\begin{tabular}{|l|c|c|}
\hline \multicolumn{1}{|c|}{ Type de burin } & Nombre & $\%$ \\
\hline Burin dièdre déjeté ou droit & 35 & 15,08 \\
Burin dièdre d'angle & 44 & 18,96 \\
Burin d'angle sur cassure & 84 & 36,2 \\
Burin dièdre multiple & 47 & 20,25 \\
Burin dièdre sur troncature & 8 & 3,44 \\
Burin de Corbiac & 6 & 2,58 \\
Burin multiple mixte & 8 & 3,44 \\
\hline Totaux & 232 & 99,95 \\
\hline
\end{tabular}

Les nombreux burins sur cassure ont donné lieu à diverses mesures statistiques. Les résultats concernant la largeur des biseaux n'ont pas paru significatifs. En revanche le diagramme unimodal concernant la distribution des mesures d'angle des biseaux semble marquer l'homogénéité de la série (fig. 10).

Figure 10 - Histogramme angle du biseau des burins d'angle sur cassure.

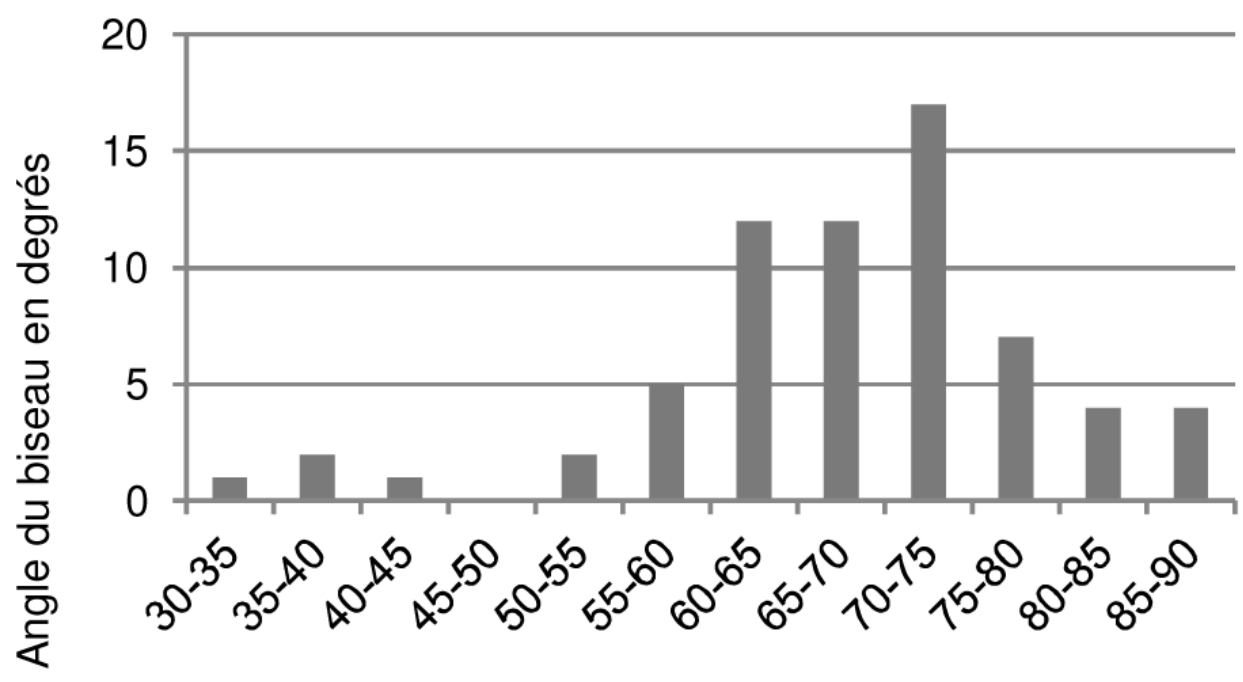

\section{Nombre d'outils}

Enfin, un burin d'angle sur cassure (fig. $9, n^{\circ}$ ) et un burin de Corbiac (fig. 9, $n^{\circ} 7$ ) présentent sur l'un de leurs bords une forte retouche inverse esquilleuse sans relation apparente avec leur fonction de burin. Les études et expérimentations concernant de telles retouches (Barton 1986) suggèrent une explication par le travail de matériaux semi-durs tels que le bois animal ou végétal. 


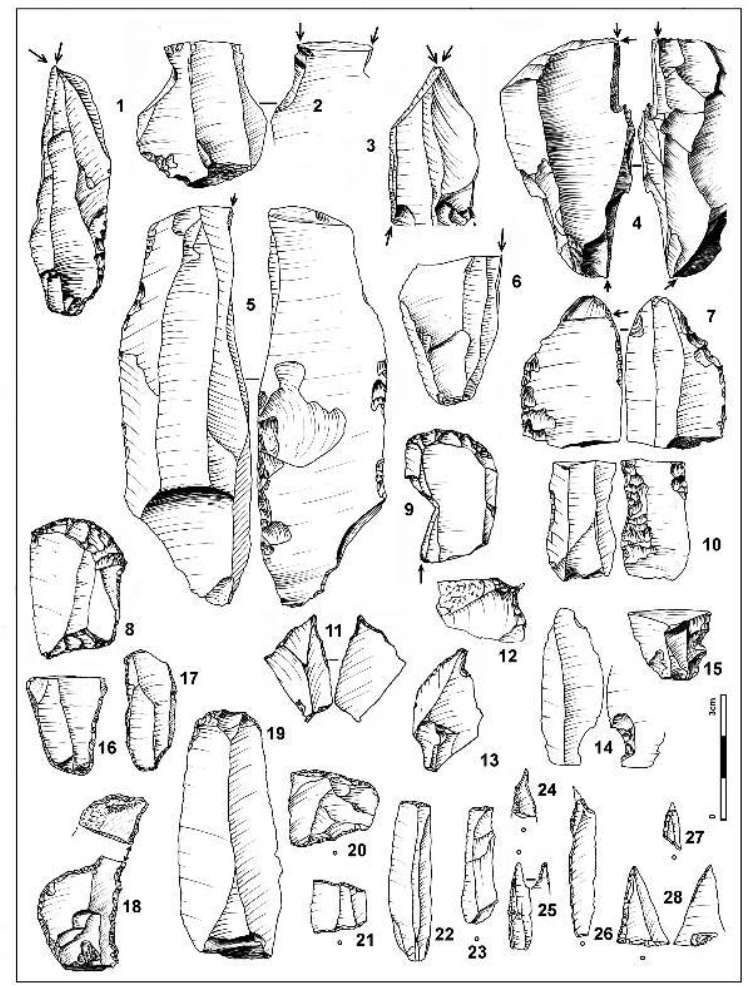

Burin dièdre, déjeté : $\mathrm{n}^{\circ} 1$. Burin dièdre multiple : nos 2-4. Burin sur cassure : nos 5-6. Burin de Corbiac : $n^{\circ} 7$. Outil composite : nos 8-9. Perçoir : nos 11-13. Lame à retouche continue : $n^{\circ} 10$. Encoche : $n^{\circ} 14$. Denticulé : $n^{\circ}$ 15. Raclette : nos 16-18. Lame tronquée : $n^{\circ}$ 19. Pièce bitronquée : nos 20-21. Lamelle tronquée : nos 20-21. Microperçoir : nos 24-26. Triangles : nos 27-28 (dessin M. Allard).

\subsection{3 - Raclettes}

La présence de 23 raclettes constitue un caractère remarquable en raison de la rareté habituelle de telles pièces hors du contexte badegoulien. La raclette (fig. 9, $\mathrm{n}^{\circ} 18$ ) façonnée sur un bord droit d'éclat formait à l'origine une sorte de bec distal qui après cassure a été abandonné tandis que la partie proximale a continué à être utilisée en particulier sur le bord gauche. Une portion intacte de l'ancienne surface de fracture a permis de réunir ces deux fragments découverts près de foyers différents.

Ces raclettes réalisées, par définition, sur éclats ou fragments de lames assez minces présentent des variabilités dimensionnelles et morphologiques comparables à celles observées dans le Badegoulien. On ne connaît pas leur usage. En l'occurrence, leur retouche abrupte et usée pourrait résulter de l'abrasion de bords de plans de frappe.

Tableau 4 - Répartition catégorielle des pièces lamellaires avec dos.

\begin{tabular}{|c|c|c|c|c|c|c|c|}
\hline \multirow[t]{2}{*}{ Lamelles retouchées } & \multicolumn{7}{|c|}{ Types de retouche } \\
\hline & \multicolumn{3}{|c|}{ Retouche à gauche } & \multirow{2}{*}{$\begin{array}{c}\text { Retouche indéterminée } \\
52\end{array}$} & \multicolumn{3}{|c|}{ Retouche à droite } \\
\hline Fragments & $\begin{array}{c}\text { Distal } \\
173\end{array}$ & $\begin{array}{l}\text { Mésial } \\
258\end{array}$ & $\begin{array}{c}\text { Proximal } \\
225\end{array}$ & & $\begin{array}{c}\text { Distal } \\
496\end{array}$ & $\begin{array}{l}\text { Mésial } \\
695\end{array}$ & $\begin{array}{c}\text { Proximal } \\
912\end{array}$ \\
\hline Total fragments & \multicolumn{3}{|c|}{656} & 52 & \multicolumn{3}{|c|}{2013} \\
\hline Pièces entières & \multicolumn{3}{|c|}{68} & & \multicolumn{3}{|c|}{129} \\
\hline Total par catégorie & \multicolumn{3}{|c|}{724} & 52 & \multicolumn{3}{|c|}{2232} \\
\hline
\end{tabular}




\subsection{4 - Les pièces lamellaires avec dos et/ou troncature} retouché. Il s'agit surtout de fragments de fines lamelles à dos entre lesquels ont été recherchés des raccords. Deux catégories sont ainsi apparues, les pièces avec dos et/ou troncature à droite et celles avec dos et/ou troncature à gauche. A l'intérieur de ces catégories, les éléments distaux, mésiaux et proximaux ont été séparés afin de simplifier la recherche d'éventuels raccords (tabl. 4). Ce dispositif mettant en évidence la suprématie numérique des dos et/ou troncatures à droite a permis la réalisation de raccords réduisant la série à 2844 pièces.

\subsubsection{1 - Les pointes avec dos et/ou troncature}

Cette série a livré 725 pointes dont environ 150 entières tandis que les autres sont fragmentaires. Chaque pièce entière ou fragmentaire étant orientée comme son support, la séparation des fragments distaux et proximaux montre que $89,5 \%$ des pointes étaient aménagées sur l'extrémité proximale plus robuste.

60 L'examen microscopique des fractures indique par ailleurs que 76,7 \% d'entre elles sont bordées, côté charnière, par une amorce de languette nommée ici "proto-languette ", résultat probable d'une percussion apicale de la pièce dans le sens axial. De plus, la pression exercée sur la charnière au stade ultime de son détachement aurait provoqué le départ de très petites éclisses appelées ici « esquillures ». Celles-ci se développent sur le bord ventral ou dorsal adjacent à la charnière. La figure 11 précise des observations réalisées entre fragments qui se raccordent. Le caractère systématique de ces fractures pourrait être lié à la finesse des lamelles. Il ne semble pas que de tels stigmates - protolanguette et esquillures - aient été observés sur des armatures plus épaisses censées avoir armé des javelots. En revanche, les lamelles fines et légères du site peuvent répondre à des armatures de flèches.

61 Sur d'autres fragments de pointes, les fractures dépourvues de stigmates de percussion pourraient en partie résulter du piétinement. 
Figure 11 - Schéma d'une fracture de lamelle avec protolanguette et esquillures, d'après observation. (dessin M. Allard).

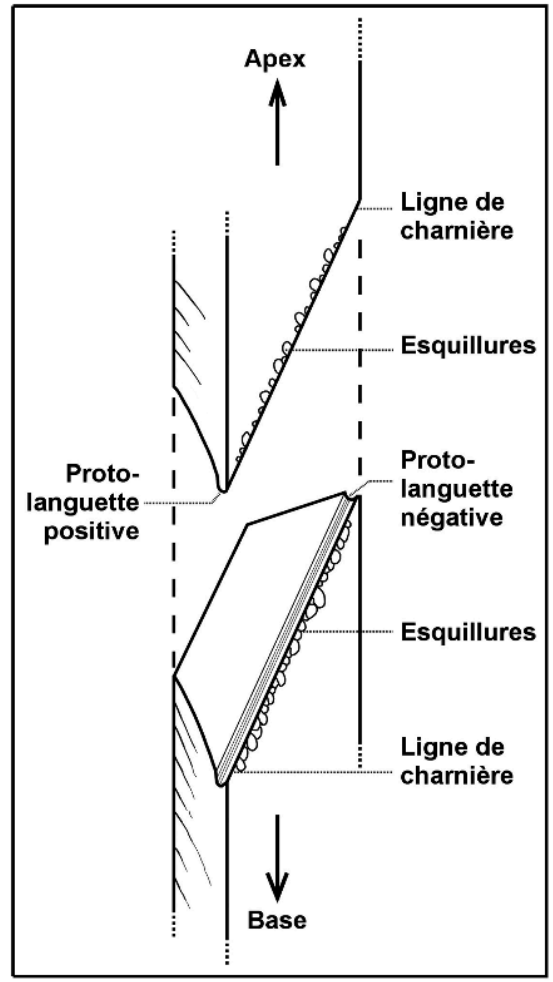

En tenant compte de ces observations, on peut établir un tableau du tri des pointes (tabl. 5) et un inventaire de leurs principaux types d'armatures rencontrés dans le gisement (tabl. 6).

Les pointes rencontrées à Auvours sont plus petites que les exemplaires équivalents rencontrés notamment sur le site des Blanchères (Rozoy 1978) (Valentin 1995). Il est possible que cette miniaturisation soit liée à la difficulté d'approvisionnement en silex ou à la petitesse des rognons.

Tableau 5 - Résultat du tri des pointes.

\begin{tabular}{|l|c|c|c|c|c|}
\hline \multicolumn{1}{|c|}{ Catégorie } & \multicolumn{2}{|c|}{ Pièces proximales } & \multicolumn{2}{c|}{ Pièces distales } & Total \\
\hline & Dos à gauche & Dos à droite & Dos à gauche & Dos à droite & \\
\hline Élément apical de pointe dont pièce & 81 & 568 & 33 & 43 & 725 \\
avec languette et/ou esquillures & $53(65,40 \%)$ & $442(77,80 \%)$ & $28(84,80 \%)$ & $33(76,7 \%)$ & $556(76,7 \%)$ \\
& & & & & \\
Pointe des Blanchères & $44(14,47 \%)$ & $239(78,6 \%)$ & $7(2,40 \%)$ & $14(4,60 \%)$ & $304(41,93 \%)$ \\
Pointe à troncature oblique & 16 & 85 & 13 & 23 & $137(18,90 \%)$ \\
Pointe indéterminée & 21 & 244 & 6 & 13 & $284(41,10 \%)$ \\
\hline
\end{tabular}

\subsubsection{2 - Des " armatures d'Auvours à apex non aménagé "}

64 La découverte de stigmates de percussion sur les pointes nous a incité à poursuivre leur recherche sur l'ensemble des lamelles retouchées. Cela a conduit, en particulier, à sélectionner un lot de 438 lamelles fragmentaires et proximales présentant près du talon un dos concave ou une encoche ayant pu servir à leur fixation (fig. 12, $n^{\circ} 43-58$ ). La retouche latéro-proximale de ces pièces forme souvent un petit éperon à sa rencontre avec le talon ce qui a pu faciliter leur fixation sur une hampe. Lorsque cette retouche ne descend pas jusqu'au talon, l'éperon peut être constitué par un reste du 
bord naturel de la lamelle (fig. 12, nos 46, 50). Enfin, dans de rares cas, le façonnage basal de l'éperon a entraîné la disparition du talon (fig. 12, nos 54, 58). 75 \% de ces fragments proximaux présentent sur leurs cassures des proto-languettes (141 cas) et des esquillures (197 cas) identiques à celles observées sur fragments basaux de pointes. Ils sont donc censés appartenir à des bases d'armatures. Par ailleurs, les quelques pièces découvertes entières, rattachables à ce type, présentent une extrémité distale naturelle fine plus ou moins tranchante.

Tableau 6 - Inventaire des principaux types de pointes déterminées.

\begin{tabular}{|l|c|c|c|c|}
\hline \multicolumn{1}{|c|}{ Types de pointe } & Nombre & $\%$ & $\begin{array}{c}\text { Pointes } \\
\text { proximales }\end{array}$ & $\begin{array}{c}\text { Dos à } \\
\text { droite }\end{array}$ \\
\hline P. des Blanchères & 304 & 55,27 & $93 \%$ & $83 \%$ \\
P. à troncature oblique & 137 & 24,91 & $74 \%$ & $79 \%$ \\
P. à base tronquée & 90 & 16,36 & & \\
P. de Petersfeld & 6 & 1,09 & $100 \%$ & $83 \%$ \\
P. de Cheddar & 4 & 0,73 & & \\
P. segmentiformes & 9 & 1,64 & $100 \%$ & $100 \%$ \\
\hline \multicolumn{1}{|c|}{ Total } & 550 & 100 & & \\
\hline
\end{tabular}

L'absence quasi systématique des parties distales tend à accréditer l'hypothèse qu'après le bris des armatures à la chasse seules leurs parties proximales auraient été rapportées au campement pour y être changées. Une exception toutefois (fig. 12, $\mathrm{n}^{\circ} 57$ ) : deux fragments de la même pièce ont été raccordés. Le fragment proximal présente des esquillures tandis que le fragment distal, pourvu d'une extrémité tranchante naturelle, a été réaménagé avec un dos avant que son apex ne soit à nouveau brisé. Il serait surprenant que de telles pièces n'existent qu'à Auvours. Il se pourrait que, considérées comme des préformes, elles n'aient pas été répertoriées. 


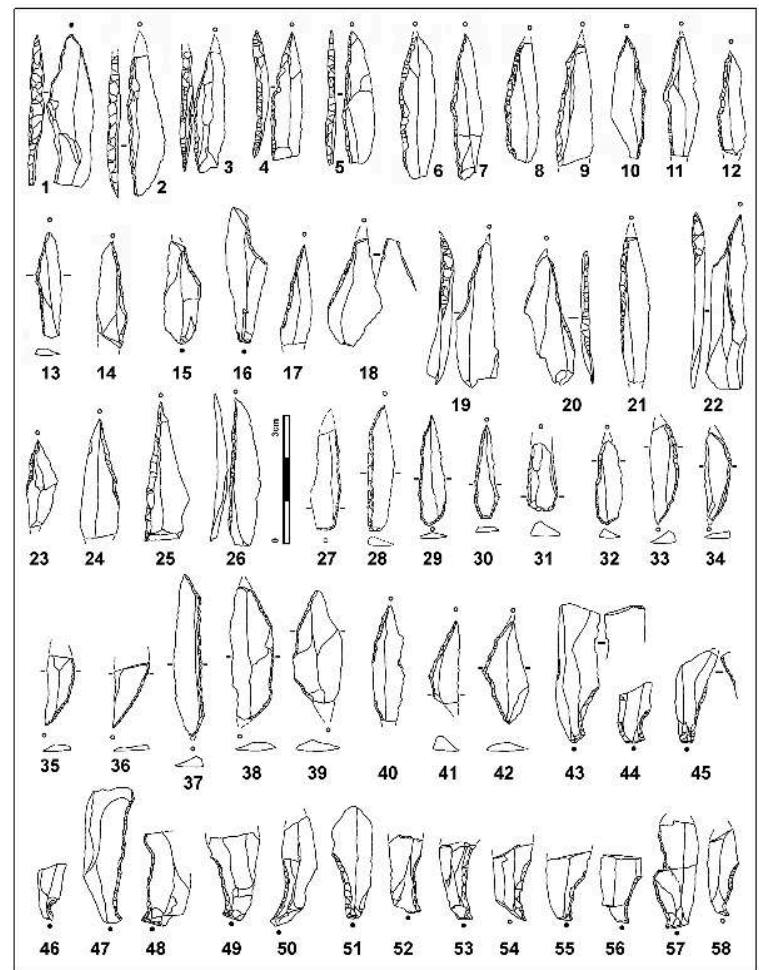

nos 1-14. Pointes à troncature oblique : nos 15-26. Pointes à base tronquée : nos 27-32 dont pointe de type Malaurie : nos 27-28 et pointe de type Istres : nos 29-32. Segments : nos 33-36. Pointes de Cheddar: nos 37-40. Pointes de Petersfeld : nos 41-42. Armatures d'Auvours : nos 43-58 (dessin M. Allard).

La fréquence de ces pièces dans le gisement tend ainsi à justifier l'appellation « armature d'Auvours à apex non aménagé » proposée dans le titre pour les qualifier. Cependant, l'étude de ces armatures mérite d'être reprise et complétée d'autant qu'elle suggère une diversification de l'activité cynégétique en direction probable de petits gibiers.

\subsection{5 - Attribution chronoculturelle}

Les résultats des études typologiques et statistiques convergent de façon remarquable. Il semblerait donc que l'outillage du site 1 d'Auvours puisse être rattaché par son style, à un Epipaléolithique, voire à des industries de transition du nord-ouest de l'Europe, plus ou moins parallèle à l'Azilien (Allard 1977- p.12). Cette attribution peut aujourd'hui être précisée. En effet, «[les] bitroncatures et le développement du principe même de la troncature pourrait être un élément culturel important dans la diagnose de ce Techno-complexe » (Naudinot 2008 - p. 264). C'est aussi ce que suggère L. Detrain: «Au vu des planches de l'industrie lithique (fig. 7, 9 et 12), l'assemblage d'Auvours semble appartenir au techno-complexe azilien, pointes à dos rectiligne. Il pourrait même avoir des affinités certaines avec le Belloisien. La pièce $n^{\circ} 21$ de la figure 9 évoque les bitroncatures rencontrées dans les industries du Dryas récent (Epilaborien, par exemple). Cela a notamment été mis en évidence lors du CPF 2010 et semble une tendance ouest européenne. » 


\section{4 - Le mobilier lithique non taillé}

\subsection{1 - Les grès rainurés}

Deux pièces rainurées en grès roussard, seules de ce type à avoir été découvertes sur le site (photo $5 \mathrm{~b}$ ), se trouvaient à l'intérieur du foyer F1. Leurs dimensions maximales atteignent $55 / 40 / 18 \mathrm{~mm}$ pour l'une et $35 / 43 / 16 \mathrm{~mm}$ pour l'autre. Ces pièces de forme semi-cylindrique présentent une face plane avec une unique et large rainure médiane orientée dans le sens d'allongement du cylindre et une autre face plus ou moins bombée. Des pièces comparables, déjà signalées par J.-G. Rozoy (1978) dans le Paléolithique final et le Mésolithique, sont plus fréquentes dans la préhistoire récente. Selon cet auteur, elles auraient pu servir à lisser des hampes de sagaies ou de flèches. Les grès rainurés d'Auvours, à faible cohésion entre les grains présentent effectivement des qualités abrasives adaptées à des matériaux de dureté telle que celle du bois. Conformément à l'hypothèse émise par J.-G. Rozoy, l'analyse de ces pièces et leur lieu de découverte suggèrent que, après avoir été chauffées pour amollir les fibres du bois, leurs faces planes ont pu être serrées l'une contre l'autre autour d'une hampe en bois afin de la lisser, la régulariser et la redresser sur toute sa longueur. Dans ces conditions, l'espace disponible entre les rainures permet le passage d'une hampe de flèche d'environ $8 \mathrm{~mm}$ de diamètre. Cette hypothèse, peut ainsi être mise en relation avec celle, déjà évoquée, d'une utilisation de l'arc sur le site.

\subsection{2 - Les billes en pierre}

La limite sud de la fouille a aussi livré, dans les carrés jointifs 2-L et 2-M, deux billes naturelles en grès roussard d'un diamètre moyen de $2 \mathrm{~cm}$ (photo 5a). À ce propos, dans son étude sur «Le Périglaciaire des environs du Mans », G. Mary précise : «Billes de grès roussard: Ce sont de petits cailloux ronds ou ovoïdes de 2 à $3 \mathrm{~cm}$ de diamètre ; leurs quartz sont usés; ils possèdent le brillant des autres roussards éolisés. " (Mary 1964 - p. 81). Leur identité morphologique et dimensionnelle ainsi que leur proximité sur le site (moins d'1 m) pourraient évoquer un usage ludique, mais il paraît toutefois difficile, dans ce contexte préhistorique, de leur attribuer une fonction particulière.

\section{5 - Le matériel ocré et sa répartition}

Ce matériau abondant sur le site n'est pas à proprement parler de l'ocre mais un mélange de sable et de limon ferrugineux concrétionné en fines plaquettes (photo 5c). Son origine géographique indéterminée pourrait se situer au sein des formations tertiaires environnantes. Bien que la couleur de ce matériau dans le gisement soit le plus souvent brun-rouge, deux échantillons (carrés 8-H, 8-D) ont conservé des parties jaunâtres tandis que cinq autres, entièrement jaunes (carrés 4-F, 10-H, 9-H, 9-E, 8-I) révèlent la présence de limonite. Sachant que la limonite évolue en hématite rouge par chauffage, la couleur brun-rouge observée sur la majorité des plaquettes ne serait a priori pas naturelle mais héritée d'un contact prolongé avec le feu. L'étude minéralogique n'ayant pas été effectuée, nous ne connaissons pas la nature précise du pigment rouge. Si, selon M.-P. Pomiès (1997), la présence de maghemite (variété d'hématite) était décelée dans les échantillons rougis, cela pourrait constituer une preuve de leur transformation par chauffage. 
Photo 5 - Mobilier lithique non taillé.
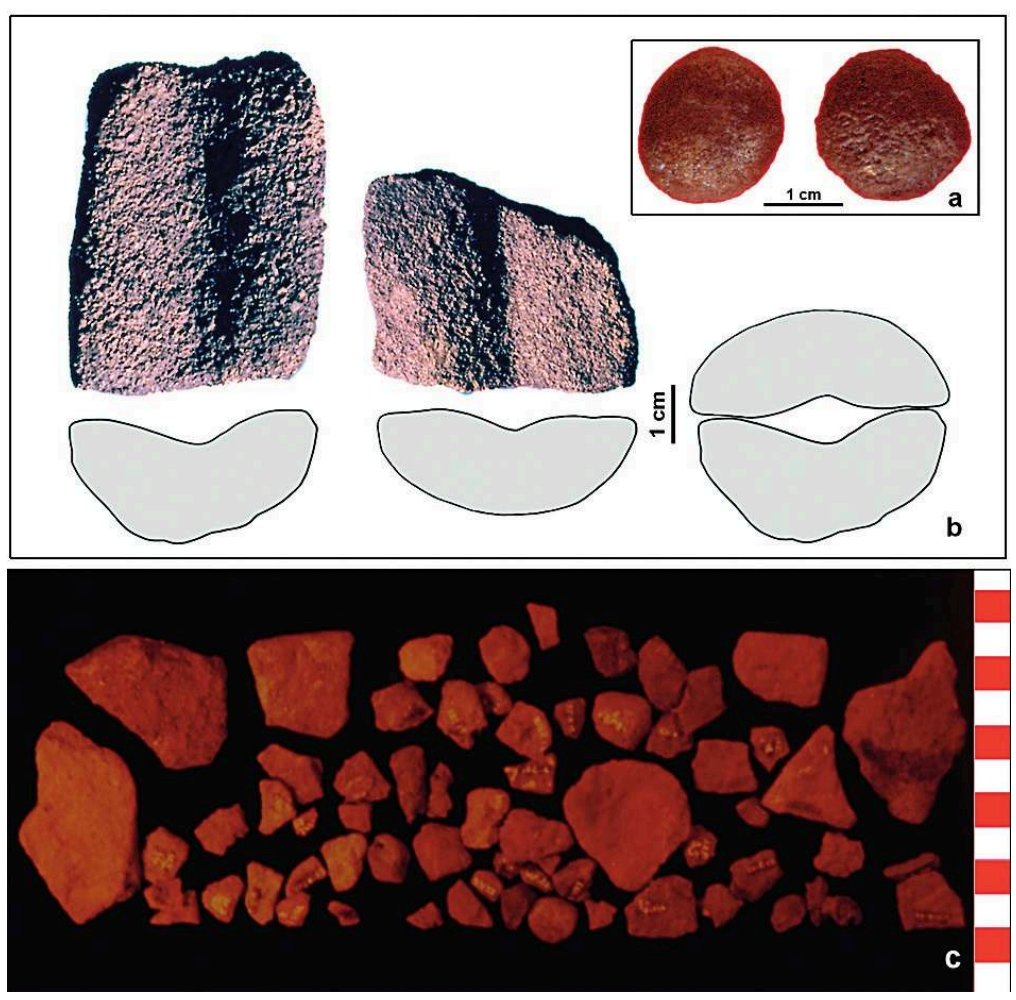

5a : Billes en pierre

$5 b$ : Lissoirs en grès

5c : Matériel ocré (cliché M. Allard).

\section{6 - Le charbon de bois et les datations ${ }^{14} \mathrm{C}$}

Les produits de combustion des foyers F1, F2 et F3 (photo 6) ne présentaient pas de charbons visibles à l'œil nu. Leur tamisage à l'eau a montré la flottation de minuscules particules charbonneuses trop pulvérulentes pour être utilement récupérables. Le mobilier lithique présent au sein de ces foyers atteste d'une attribution chronoculturelle correspondant au post-Azilien.

En revanche, la partie nord du site a livré dans les carrés 11-F et 12-F de nombreux charbons de bois directement prélevés dans une couche de combustion de 12 à $15 \mathrm{~cm}$ d'épaisseur, en forme de cuvette, comportant deux niveaux et située sous la couche archéologique post-Azilienne (cf. § 4.4.6).

73 Les analyses C14 réalisées par le laboratoire de Gif-sur-Yvette sur les charbons recueillis ont donné les résultats suivants :

- niveau supérieur (6,88 g prélevés en 11-F et 12-F), $6600 \pm 160$ ans BP (Gif., 3989)

- niveau inférieur (23,9 g prélevés en 12-F), $6460 \pm 160$ ans BP (Gif., 3990)

Ces datations attestant d'une postériorité au regard de la couche archéologique postazilienne indiquent une inversion stratigraphique. Celle-ci, au vu de la forme concave de la couche charbonneuse, pourrait s'expliquer par la présence d'un foyer profond, postérieur au Post-Azilien, creusé au travers de la couche archéologique post-azilenne 
dont les parois se seraient progressivement affaissées recouvrant ainsi la couche charbonneuse par le mobilier lithique post-azilien.

Photo 6 - Assemblage photographique légendé du site préhistorique d'Auvours (cliché M. Allard).

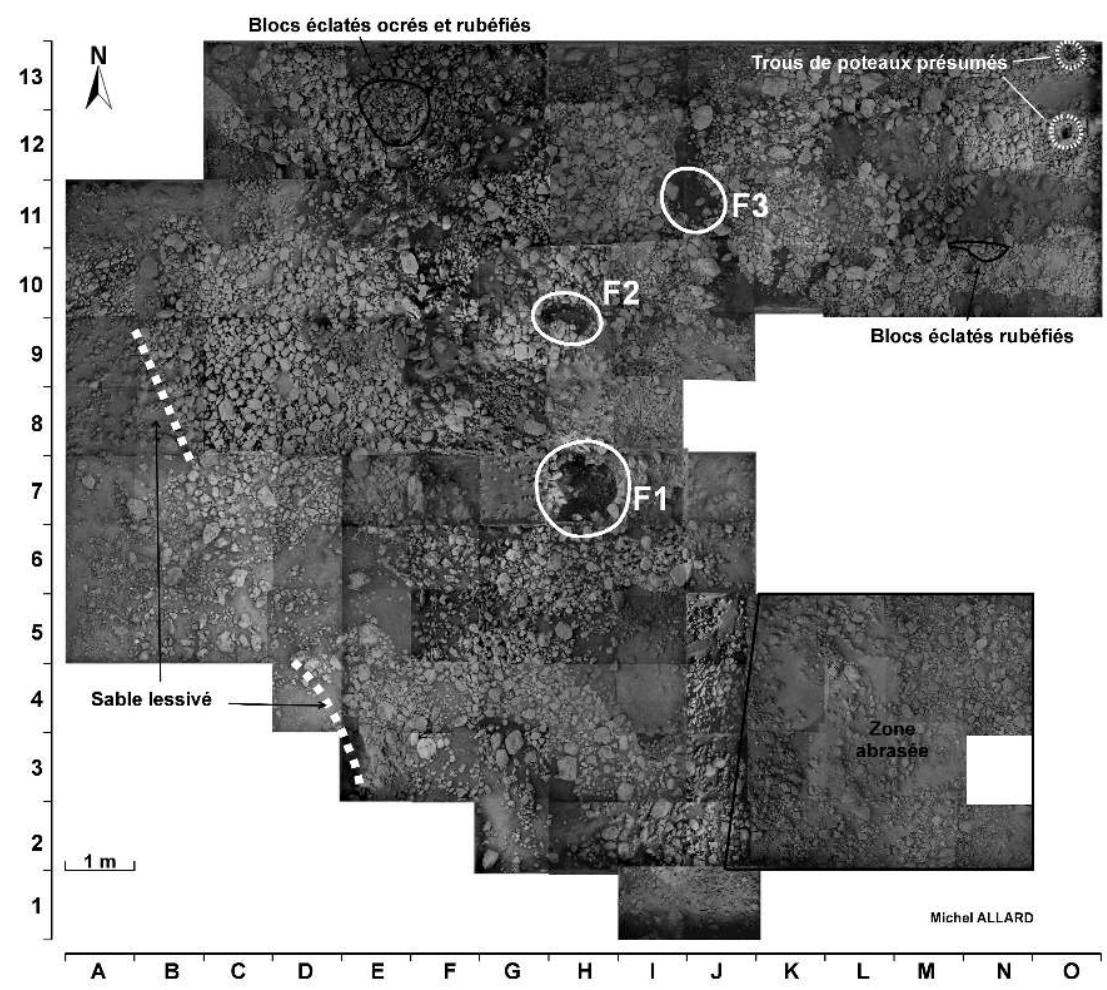

\section{4 - Les éléments structurés du site 1 d'Auvours}

\section{1 - Considérations générales}

En 1972, la première campagne de fouille a mis au jour un foyer F1 riche en mobilier archéologique, construit avec un entourage de blocs gréseux. Cette découverte essentielle a déterminé la poursuite de la fouille dont chaque carré a fait l'objet de prises de vues verticales, montrant les blocs visibles en place (photo 6).

L'assemblage photographique présente l'ensemble de la surface fouillée sur laquelle les blocs constituent diverses accumulations.

A l'aide d'observations réalisées durant la fouille et d'éléments iconographiques appropriés (photos, plans, coupes), nous nous efforcerons d'apporter les éléments indispensables à la compréhension à cet assemblage photographique. Il faut noter que les sites 2, 3, 4 et 5 ont paru dépourvus de blocs. Il semble donc que les nombreux blocs présents sur le site 1 aient été déterminants pour le choix de son emplacement. En effet, les occupants préhistoriques de ce site se seraient installés ailleurs si l'endroit ne leur avait pas convenu. 
Photo 7 - Foyer F1 en cours d'étude et de démontage de ses structures, en 1972 (cliché M. Allard).

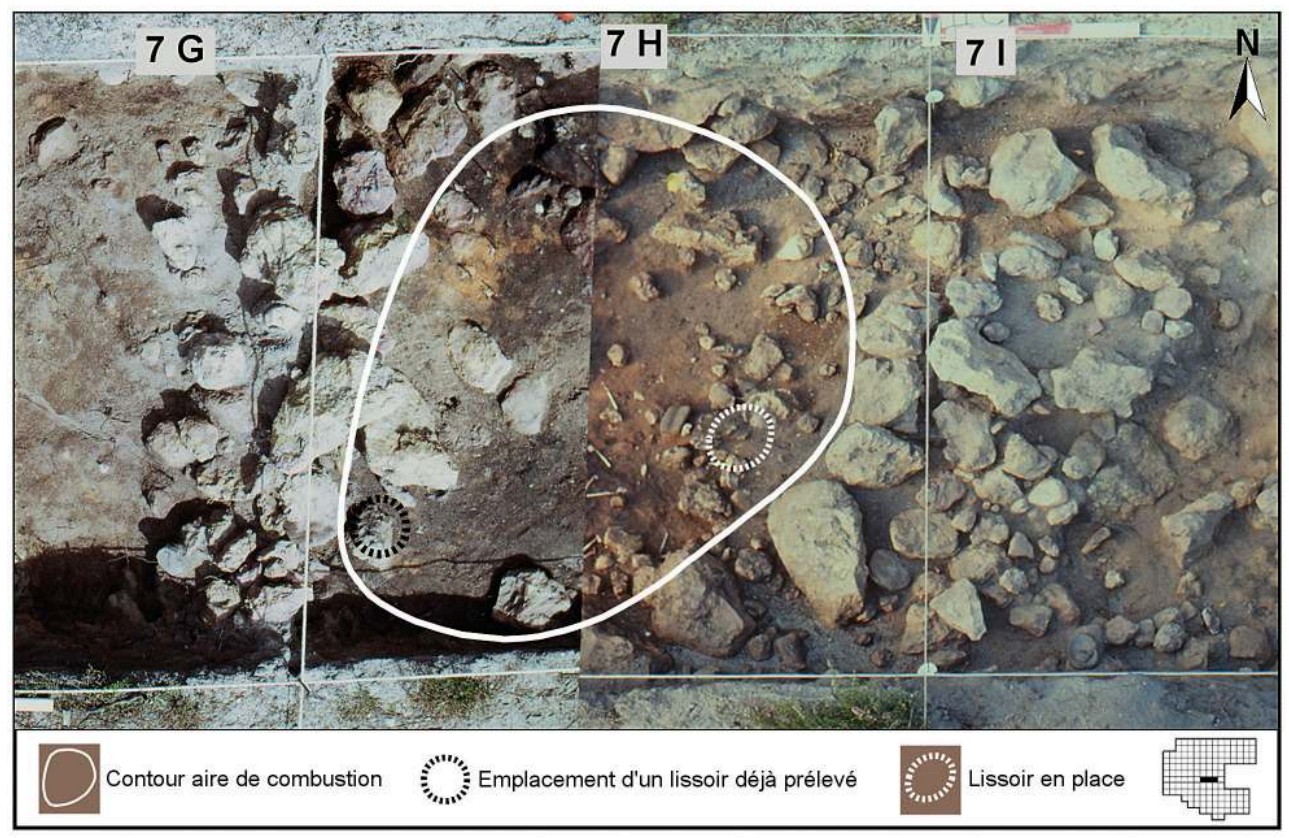

\section{2 - Analyse des foyers structurés fouillés en totalité}

78 A la suite de la découverte du foyer $\mathrm{F} 1$, la poursuite de la fouille a permis la mise au jour de deux autres unités de combustion nommées F2 et F3. L'identification de ces foyers a reposé sur plusieurs critères: leur organisation, les produits de combustion qu'ils contenaient ou qui s'en échappaient et leurs éventuels effets thermiques sur le milieu. Ils ont été entièrement fouillés.

\subsection{1 - Le foyer F1}

La structure de combustion $\mathrm{F} 1$, fouillée et démontée dès la première année, occupe une position presque centrale à l'intérieur de la zone étudiée (photo 7). Elle est inscrite dans le carré $7 \mathrm{H}$ qui a livré 4652 silex dont 74 retouchés. Son espace de combustion de $75 \mathrm{~cm}$ de diamètre était entouré par une couronne de blocs d'environ $35 \mathrm{~cm}$ d'élévation. A l'extérieur, dans le carré 7-G, un espace dépourvu de blocs témoignait de la parfaite stabilité de la bordure du foyer de ce côté. Le bord opposé s'élevait sur une assise de blocs oblongs disposés de façon rayonnante par rapport au centre du foyer. Les extrémités centripètes de plusieurs blocs étaient rubéfiées. Quelques uns découverts à l'intérieur du foyer ont semblé être versés de sa périphérie. D'autre part, à $40 \mathrm{~cm}$ environ de la surface du sol, ont été remarquées, sous la couche de combustion de $15 \mathrm{~cm}$ d'épaisseur, quelques auréoles brunes signalant l'approche en profondeur de l'horizon BPh du podzol. Le foyer 1 ainsi que la couche archéologique étaient donc entièrement inclus dans l'horizon E éluvial. Une partie des produits de combustion débordait vers le nord-ouest du foyer à la faveur d'un infléchissement de sa couronne de blocs. Sa partie interne beaucoup plus riche en mobilier que sa partie externe a livré, à $30 \mathrm{~cm}$ de profondeur, les deux seuls lissoirs en grès rencontrés sur le site (photo $5 \mathrm{~b}$ ). Enfin, côté sud, la structure de combustion était adossée à un amoncellement de blocs de trois mètres de longueur orienté ouest-est. Une reconstitution de ce foyer serait 
envisageable à partir de ses éléments conservés au musée du Mans et des relevés qui en ont été effectués.

Photo 8 - Foyer F2 avec sa cuvette centrale entourée de blocs et son canal de vidange (cliché M. Allard).

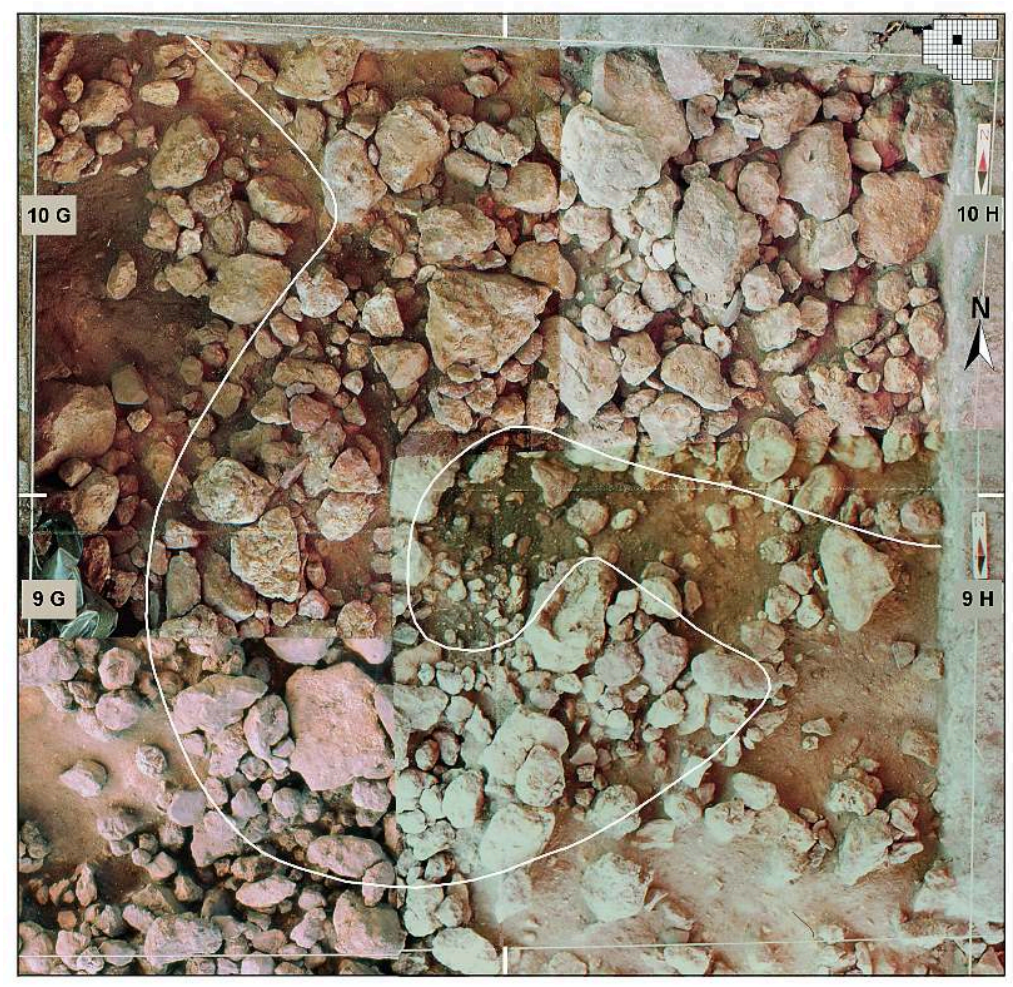

\subsection{2 - Le foyer F2}

Le foyer F2, d'environ $40 \mathrm{~cm}$ de diamètre, situé au centre des carrés 10-G, 10-H, 9-G, 9$\mathrm{H}$, était perché sur une butte sableuse adossée du côté est à un imposant amoncellement de blocs (photo 8). La partie centrale du foyer formait une petite cuvette emplie de produits de combustion de couleur noire sur une épaisseur de $5 \mathrm{~cm}$. Elle était entourée de blocs, sauf du côté est ouvert sur une sorte de goulotte par où s'échappaient des produits de vidanges de même couleur. La base de la cuvette se situait entre 25 et $30 \mathrm{~cm}$ sous la surface du sol. Avec près de 12000 silex dont 118 outils au mètre carré (fig. 5), la bordure orientale de ce foyer a constitué le secteur de la fouille le plus riche en mobilier. Côté ouest et nord-ouest, sa butte était bordée par un creusement profond d'une quinzaine de centimètres, large d'environ $1 \mathrm{~m}$. Au niveau du carré 9-F, ce creusement formait une sorte de chenal dont le contenu, de couleur grisâtre, était constitué de cendres mêlées au sable. La coupe du foyer et du chenal précise ces données (fig.13). 
Figure 13 - Coupe du foyer F2. Sa bordure ouest dans le carré 9-F est marquée par un creusement qui a constitué une sorte de chenal suivi vers le nord jusqu'à l'accès P2 (dessin M. Allard).

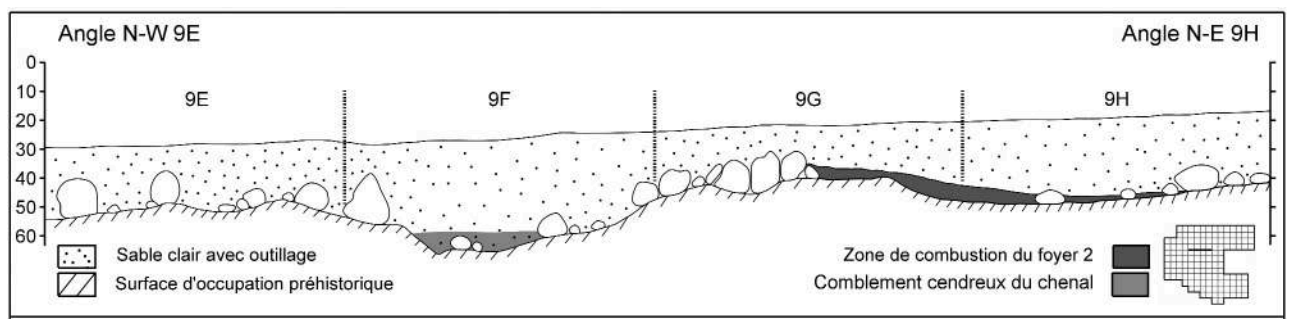

\subsection{3 - Le foyer F 3}

81 Au centre des carrés 12-I, 12-J, 11-I, 11-J, le foyer F3 formait une cuvette de $60 \mathrm{~cm}$ de diamètre (photos 9 et 10), contenant une couche de combustion de $10 \mathrm{~cm}$ d'épaisseur, très sombre. Cette zone a livré 30347 silex dont 401 retouchés (fig. 8). Le foyer se prolongeait vers le nord par un long évent dont l'extrémité resserrée entre deux blocs volumineux débouchait dans le carré 13-J. Les produits de combustion expulsés par cet évent ont livré 7204 silex dont 72 retouchés dans le carré 13-J.

Photo 9 - Foyer F3. Vue verticale générale obtenue par assemblage photographique (cliché M. Allard).

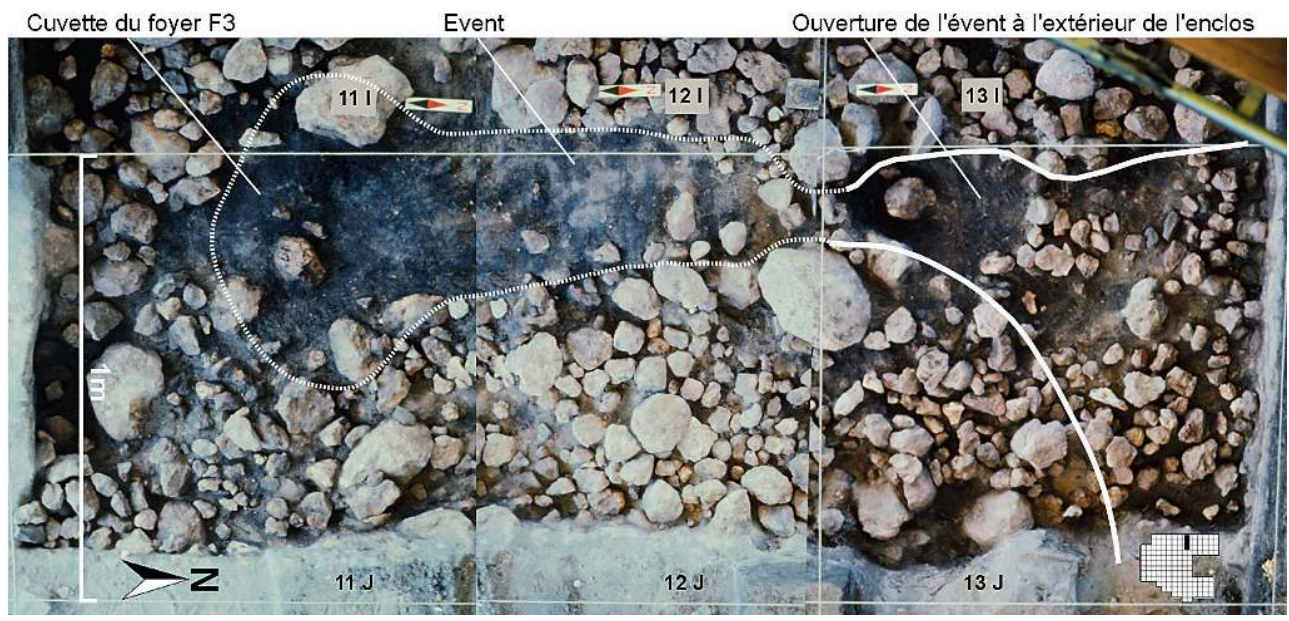

Le foyer et son évent, installés au cœur d'une vaste et épaisse accumulation de blocs, présentaient un fond sableux noirâtre et induré à $50 \mathrm{~cm}$ sous la surface du pare-feu. À cette profondeur, la base du foyer, en creux par rapport aux empierrements latéraux, rejoignait l'horizon BPh noir du podzol. Si une partie des produits de combustion a été expulsée par l'évent, la plus grande partie de ces produits était répandue depuis le foyer jusque dans les carrés 10-J, 9-J. Ce secteur a livré de nombreux témoins ocrés ainsi qu'un mobilier lithique totalisant 15974 silex dont 180 retouchés. 
Photo 10 - Secteur du foyer F3 vu de l'est. Ce cliché offre un aperçu du relief des accumulations de blocs (cliché M. Allard).

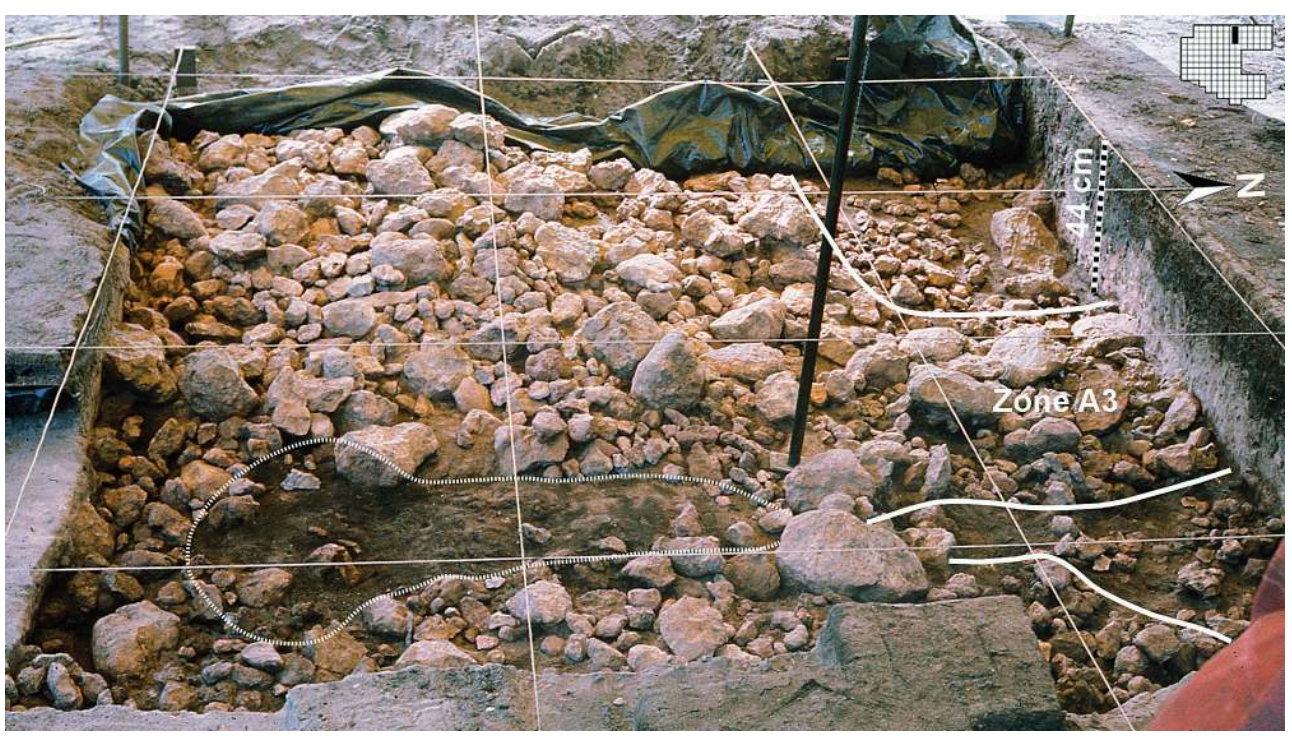

\subsection{4- Conclusion sur les foyers F1, F2, F3}

Les foyers F1 à plat et F2 perché sur une butte sableuse étaient délimités chacun par une couronne de blocs. Par contre, le foyer F3 avait été creusé jusqu'à la couche noire du podzol au sein d'une importante accumulation de blocs y formant une cuvette prolongée par un évent. Les produits de combustion noirâtres et meubles de ces foyers riches en mobilier lithique ont montré par flottation, lors du tamisage, de nombreuses mouchetures charbonneuses tandis que le sédiment noir et induré du podzol observé à la base de F3 s'est montré à cet égard parfaitement stérile.

Photo 11 - Vue de la partie nord-est de la fouille à partir de sa bordure est (cliché M. Allard).

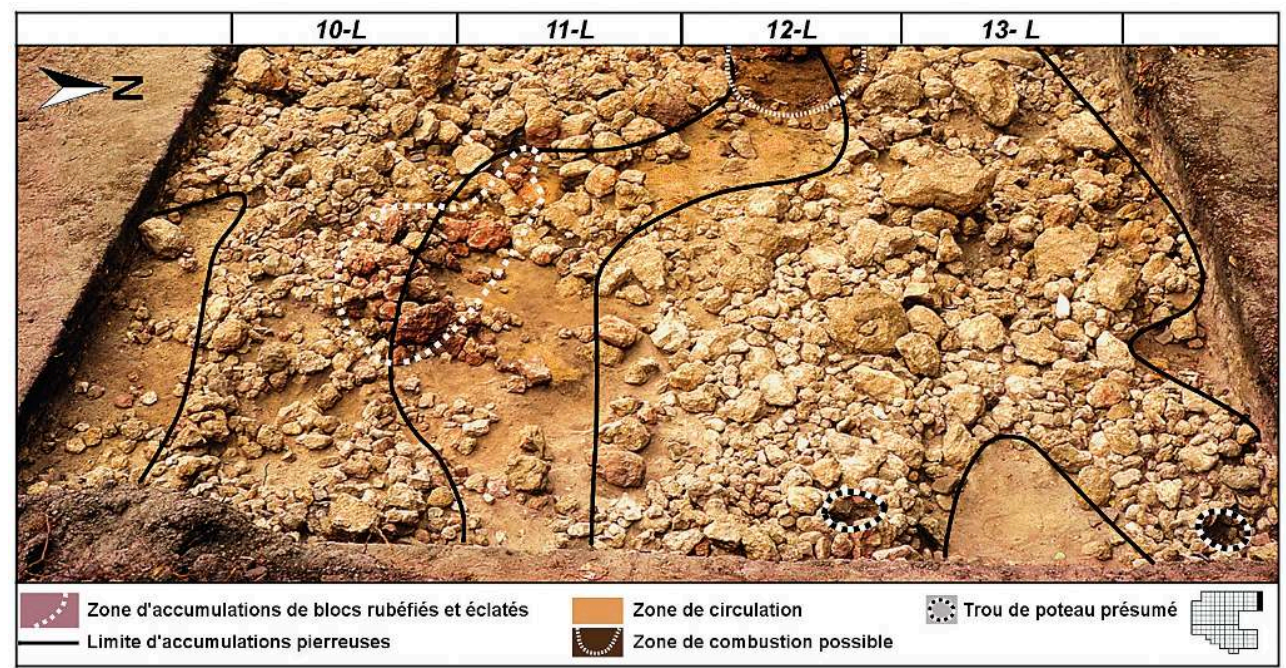

84 L'abondance des produits débités aux abords des foyers suggère une importante activité de taille et de retouche du silex. La présence de mobilier à l'intérieur des foyers pourrait résulter de prélèvements de sable effectués à leurs abords puis jetés sur les braises pour en ralentir la combustion. Cette hypothèse déjà évoquée (Allard 1982) 
permettrait d'expliquer, à la fois l'abondance du mobilier lithique à l'intérieur des foyers et la fréquence des effets thermiques observés sur celui-ci. Les silex situés dans les zones de vidanges présentent les mêmes caractéristiques thermiques.

Photo 12 - Trou de poteau présumé situé dans le carré 12-0 (cliché M. Allard).

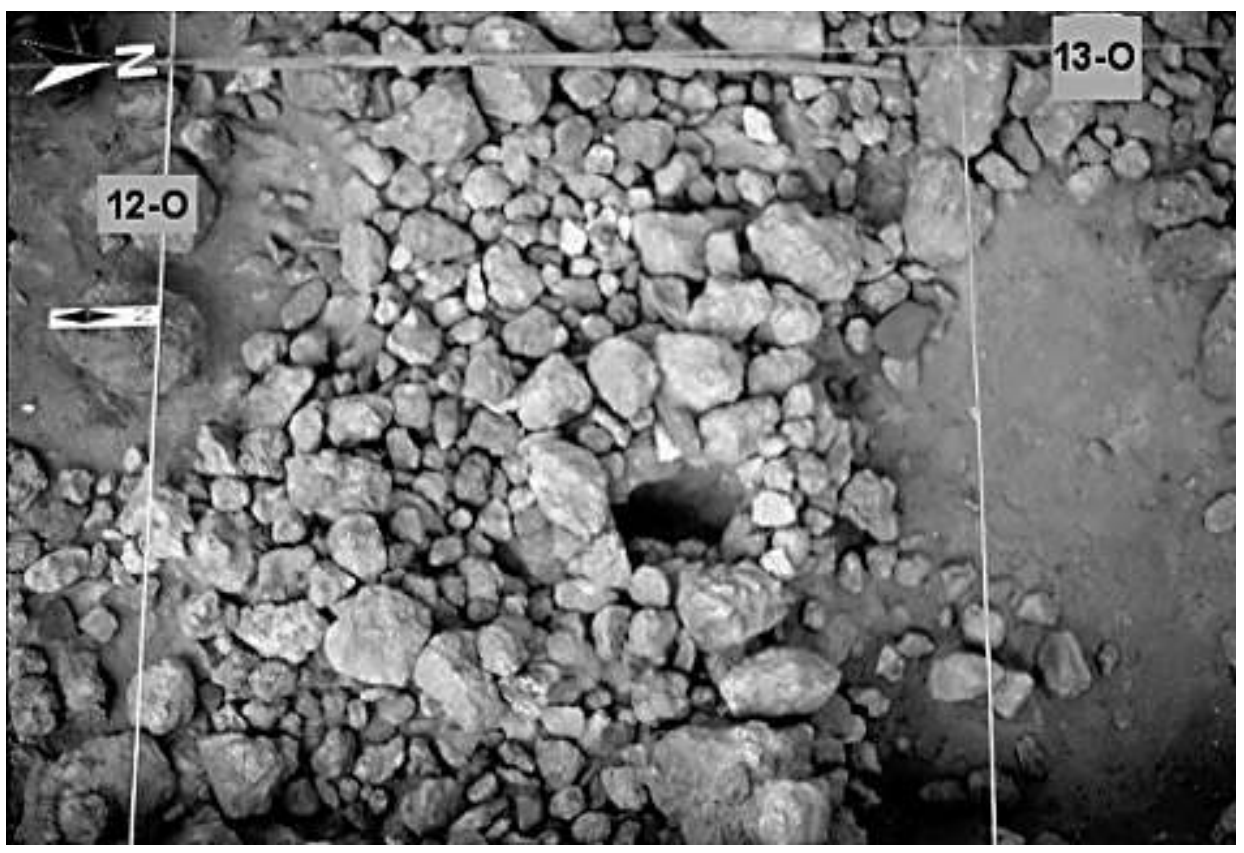

La richesse du mobilier (327 000 silex) ainsi que la présence de trois foyers structurés dont l'épaisseur des couches de combustion varie de 5 à $15 \mathrm{~cm}$ d'épaisseur témoignent d'une installation humaine de longue durée sur ce site.

\section{3 - Les trous de poteaux présumés du secteur nord-est}

Dans l'angle nord-est (carré 13-0) une ligne de blocs, aux bords francs, présentait à son sommet une cavité circulaire de 6 à $7 \mathrm{~cm}$ de diamètre. De petits blocs $(<5 \mathrm{~cm})$ bordant cette cavité constituaient un léger bourrelet à sa périphérie sommitale. Explorée sur 8 $\mathrm{cm}$ de profondeur, elle n'a livré qu'un sédiment sableux de même coloration que le reste de l'horizon $\mathrm{E}$ du podzol dans lequel il ne subsiste pas de traces d'humus. Bien que 1123 silex aient été trouvés dans ce carré, la cavité n'en a fourni aucun. Une cavité similaire étudiée dans les mêmes conditions a été rencontrée dans le carré 12-0 au sommet d'une autre ligne de blocs (photos 11 et 12). Interpellé par l'intérêt de ces vestiges, nous avions décidé de les conserver momentanément en l'état, prévoyant de poursuivre l'exploration de leur cavité dans le cadre d'une réouverture générale du site, ce qui n'a pas pu être réalisé.

Bien que le fond de ces deux cavités n'ait pas été atteint, il n'en reste pas moins que la verticalité de leurs parois internes et la bonne tenue des blocs qui les constituaient présument l'aménagement de calages de poteaux verticaux. 


\section{4 - Observations complémentaires}

F) a montré la présence de mobilier lithique au sommet de cette accumulation, mais l'absence de mobilier dans sa partie interne. Cette observation a été déterminante pour la suite de la fouille où les massifs de blocs ont été conservés indemnes afin d'en permettre une vision d'ensemble.

\subsection{1 - Hypothèse de cryoturbation}

La présence massive de blocs et de foyers structurés sur le site 1 d'Auvours nous a interrogé sur l'origine de ces amas de blocs. Leur constitution était-elle naturelle ou avaient-ils été aménagés? Ce questionnement nous a conduit à des inspections attentives du terrain. En particulier, nous avons pu observer deux coupes longues d'une vingtaine de mètres et de plus plus d'un mètre d'épaisseur dans la partie supérieure de la basse terrasse. La première longeait la bordure nord du pare-feu entre le site 1 et la retombée de la terrasse sur le lit majeur du Narais; la seconde, sectionnant le site 3 au sud de la piste, résultait d'un décaissement de la parcelle 291 (fig.1-b) en 1979. Ces coupes, réalisées hors de toute zone de pavement dans une formation essentiellement sableuse n'ont montré ni figures de cryoturbation ni fentes en coin. En outre, les nombreuses coupes, réalisées dans la fouille ainsi que les observations en plan effectuées depuis la surface du pare-feu jusqu'au fond de la zone fouillée n'ont pas davantage révélé d'indices de cryoturbation. Le géologue G. Mary spécialiste et bon connaisseur du Quaternaire de ce secteur nous a redit récemment, en 2013, qu'aucune cryoturbation ni fente en coin qui affectent les hautes et moyennes terrasses des alentours (Mary 1964 - p.76) n'avait été signalée dans la basse terrasse. En conséquence, l'hypothèse de l'accumulation par cryoturbation des blocs du site 1 , qu'aucun argument ne vient étayer, n'a pas lieu d'être retenue.

Par ailleurs, la structuration élaborée de chaque foyer telle que la disposition rayonnante des blocs à la périphérie du foyer 1 , l'installation du foyer 2 dans une cuvette cernée de blocs au sommet d'une petite butte, l'aménagement profond du foyer 3 au cœur d'un massif de blocs ne résultant pas de phénomènes naturels, atteste d'un aménagement anthropique sur ces zones de combustion.

\subsection{2 - Le secteur sud-est}

91 Le secteur sud-est de la zone fouillée, affecté par un décapage plus profond du pare-feu en bordure de la piste, s'est avéré peu exploitable sur le plan archéologique, en particulier dans les bandes KLMN où l'horizon $\mathrm{E}$ du podzol contenant les blocs avait partiellement disparu.

\subsection{3 - Le secteur nord-est}

Dans le secteur nord-est, (bande KLMNO), la photo 6 complétée par la photo 11, montre des amas pierreux linéaires séparées par des espaces appauvris en blocs. On y distingue :

93 - dans l'angle nord-est (carré 13-0) une ligne de blocs, aux bords francs, large d'environ $40 \mathrm{~cm}$, haute de $20 \mathrm{~cm}$, présentant à son sommet un trou de poteau présumé. Cette 
bande rejoint une accumulation linéaire de blocs plus conséquente dans les carrés $12-\mathrm{N}$ et 12-0. Cette dernière offre un flanc nord nettement vertical. La présence d'un autre trou de poteau semblable au précédent dans le carré $12-0$ à moins de $10 \mathrm{~cm}$ de ce flanc nord témoigne de la remarquable stabilité de l'ensemble. En revanche, la bordure sud de cet amas de blocs est en partie éboulée ;

94 - au sud de ces accumulations, les carrés 11-N et 11-O sont traversés par une une zone d'1 $\mathrm{m}$ de large, très appauvrie en blocs. Celle-ci se prolonge de façon sinueuse jusque dans le carré 12-L où le sol noirci semble correspondre à l'emplacement d'un foyer détruit. Des résidus de combustion noircis associés à des produits ocrés y sont étalés depuis le carré 12-L jusque dans le carré 11-N bordé côté sud par un groupe de blocs éclatés et rubéfiés. Cette zone dégarnie de blocs, plus riche en mobilier préhistorique que ses abords immédiats, semble marquer une zone de circulation. Celle-ci se prolonge dans les carrés $11-\mathrm{K}, 11-\mathrm{L}$ et 10-K, 10-L entre deux accumulations de $25 \mathrm{~cm}$ de haut, dont les flancs sont légèrement éboulés (photo 6).

\subsection{4 - Le secteur sud-ouest}

Deux accumulations pierreuses hautes de 20 à $25 \mathrm{~cm}$ et de $10 \mathrm{~m} 2$ environ chacune, s'étendent entre les carrés $11-\mathrm{B}$ et 2-J sur une longueur de $11 \mathrm{~m}$. On y remarque :

96 - la zone comprise entre les carrés 10-BCDE et 7-BCDE, présente une accumulation de blocs massive, de forme complexe, longue de $5 \mathrm{~m}$ et d'une largeur moyenne de $2 \mathrm{~m}$. Au sein de celle-ci, trois petits espaces de 10 à $15 \mathrm{~cm}$ de diamètre, sans forme caractéristique mais remplis de sable - carrés 10-C, 9-D et 7-E - alignés et régulièrement espacés d'un mètre dans la zone axiale n'ont fait l'objet d'aucune recherche approfondie. En 7-B et 6-B, un amas de blocs, moins dense et moins élevé de 10 à $15 \mathrm{~cm}$ de haut - marque un décrochement perpendiculaire à l'axe d'allongement général ;

97 - la zone comprise entre les carrés 4-EFGH et 2-IJ comporte une accumulation de blocs sinueuse, longue de $6 \mathrm{~m}$, large d'1 $\mathrm{m}$ et haute de 20 à $25 \mathrm{~cm}$. Sa plus grande longueur ( 4 m) se situe dans le même axe que les alignements observés dans l'accumulation précédente. Son extrémité (carré 4-E essentiellement) forme un décroché en L orthogonal à l'axe principal et également élevé de 10 à $15 \mathrm{~cm}$;

98 - les carrés 4-E, 4-D et 3-E, en limite de l'accumulation de blocs ont été marqués par la présence d'un sable blanc dépourvu d'enduit limoneux dont la granulométrie grossière correspondait aux caractéristiques d'un sable lessivé. Sur une distance d'un peu plus d'1 $\mathrm{m}$, celui-ci marquait une dépression profonde de 3 à $4 \mathrm{~cm}$ et large de $20 \mathrm{~cm}$. Une observation identique a été réalisée sur une distance de $2 \mathrm{~m}$ environ dans les carrés 9A, 9-B et 8-B en limite de l'accumulation de blocs (photo 6);

99 - la partie intermédiaire, carrés 6-CDEF et 5-CDEF, située entre les deux accumulations précédemment décrites comporte une bande longue de $4 \mathrm{~m}$ et large de près de $2 \mathrm{~m}$, appauvrie en blocs ;

100 - la diminution quantitative du mobilier lithique à l'ouest de ces deux accumulations de blocs (fig. 8) suggère une limite de la zone d'installation. 


\subsection{5 - Le secteur nord-ouest}

101 - $A u$ niveau des carrés 11-ABC, une zone appauvrie en blocs présentant un rétrécissement en 11-DE se prolonge de façon sinueuse jusque dans les carrés 13-GH. Sa distinction sur le terrain facilitée par le relief est en revanche plus délicate sur l'assemblage photographique (photo 6)

102 - En bordure nord de cette bande sinueuse, dans la zone 12-CDE, 13-D, une accumulation de blocs, longue de 2,50 m, haute de $30 \mathrm{~cm}$ en 12-E et de $20 \mathrm{~cm}$ en 13-D, se divise en deux branches d'inégale largeur orientées vers l'angle nord-ouest de la zone fouillée. Sur son flanc est s'appuie un amas exclusivement constitué de blocs éclatés, ocrés et rubéfiés, d'environ $1 \mathrm{~m} 2$.

103 - Dans les carrés 11-F, 12-F et 12-G le sédiment sableux est fortement ocré. Sa teinte rose saumon ponctuée de taches charbonneuses s'atténue progressivement vers le nord de la zone fouillée (carrés 13-EFGH). La partie sud de ce secteur, la plus colorée, a livré un grand nombre de plaquettes et de granules ocrés. Elle s'est aussi montrée particulièrement riche en mobilier lithique (> 7500 vestiges au $\mathrm{m} 2$, fig. 5 ).

104 - Côté sud, la zone appauvrie en blocs est délimitée par deux accumulations, l'une décrite en début de paragraphe 4.4.4., l'autre d'environ $3 \times 4 \mathrm{~m}$ formant un dôme, incluant le foyer F3 et bordée au sud-ouest par le foyer F2. Cette limite est marquée en 13-H, 12-G et 11-F par des décrochements soulignés sur le terrain par le relief abrupt des accumulations voisines hautes de $30 \mathrm{~cm}$. Des décrochements comparables se retrouvent en 11-CDE.

\subsection{6 - Observations relatives au secteur nord-ouest du foyer F2}

105 Le secteur compris entre le foyer F2 et le carré 11-F a été fouillé une première fois jusqu'au niveau des accumulations de blocs laissées en place (fig. 14). Cette opération a mis au jour, en 9-F et 10-FG, une bande grise cendreuse plus profonde. Son dégagement ultérieur a montré que les cendres comblaient un chenal large d' $1 \mathrm{~m}$ et profond d'une quinzaine de centimètres dans sa partie centrale. Il a été suivi depuis la butte du foyer F2 jusqu'à la limite sud du carré 11-F. À cet endroit, nous avons observé une modification du contenu du chenal, devenant plus compact et plus sombre. Ainsi est apparue, à sa base, une couche formée de deux niveaux, riche en produits charbonneux, s'épaississant vers le nord de façon à atteindre rapidement $15 \mathrm{~cm}$. Le niveau supérieur très induré, de 5 à $6 \mathrm{~cm}$ d'épaisseur, contenait quelques petits charbons de l'ordre du millimètre. Le niveau inférieur marron, meuble, sensiblement plus épais, contenait de nombreux charbons proches parfois d'1 cm3. Leur datation est commentée au § 3.6. 
Figure 14 - Coupe 10-E, 11-G (dessin M. Allard).

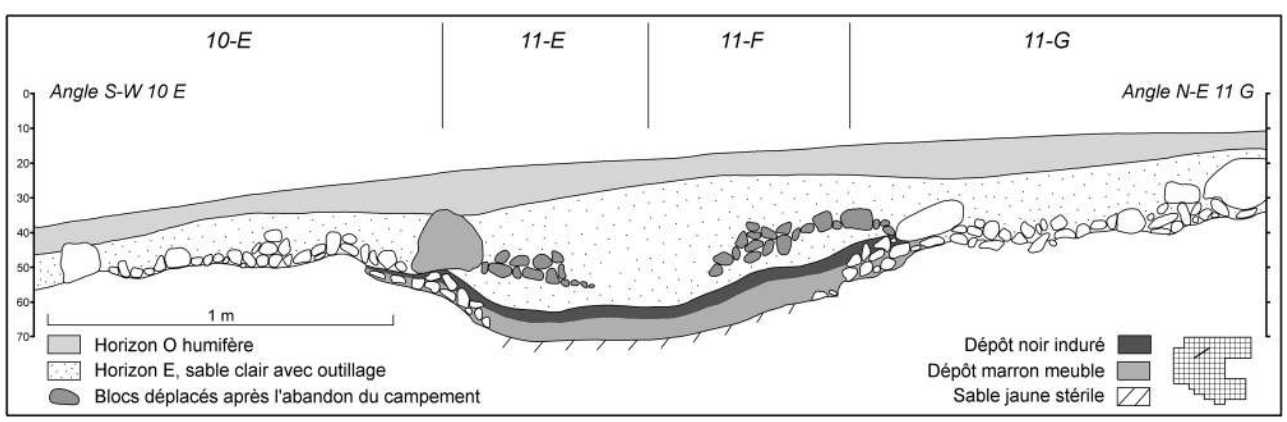

\section{5 - Relations entre structures et mobilier préhistorique}

Bien que la fouille du site 1 d'Auvours n'ait pu être menée à son terme, la recherche archéologique a permis d'établir des relations significatives entre ses éléments structurés et son mobilier.

\section{Limites du gisement}

Les sondages ont livré des informations sur les limites probables du gisement vers l'ouest (S4, S5), le sud (S6) et l'est (S2, S3). Le sondage S1 réalisé dans la lande au nord du pare-feu a révélé le développement du site dans cette direction. La fouille a confirmé et précisé ces indications malgré les effets de l'érosion depuis l'abandon préhistorique du site. Par ailleurs, la diminution graduelle du nombre d'objets marque d'une part une zone d'activité intense autour des foyers et souligne d'autre part la présence de la longue accumulation pierreuse barrant la partie sud-ouest de la zone fouillée (fig. 5).

\section{Répartition du mobilier}

L'ensemble du mobilier (327 000 silex) était réparti essentiellement dans le quart nordest de la fouille autour d'espaces de combustion près desquels ont été trouvées d'importantes concentrations de déchets de taille. Cependant, l'étude de la distribution du mobilier lithique (nuclei, grattoirs, burins, grès débités, plaquettes ocrées, pointes, fragments lamellaires avec dos et liaisons entre fragments débités) ne montre pas de regroupements catégoriels significatifs (fig. 15abc). Une exception toutefois, deux plaquettes de grès rainurés ajustables ont été découvertes à soixante centimètres l'une de l'autre dans le foyer F1.

\section{Présence de plaquettes ocrées et d'ocre}

La coloration brun-rouge des plaquettes ocrées indique que celle-ci a été acquise par chauffage (photo 5c). Sans exclure l'hypothèse d'une utilisation rituelle de l'ocre peinture corporelle, teinture des parures et des armes, dessins sur peaux ou écorces, sépultures (Bordes 1952) (Triat 2011) - encore pratiquée par de nombreux groupes humains (Leroi-Gourhan 1955, 1976), il semble qu'ici la quantité considérable de pigment dans le sol a dû correspondre à l'existence d'un atelier de broyage des matériaux ocrés et/ou éventuellement d'une station de tannage employant l'ocre pour 
le traitement des peaux. Ce fait concorde avec la présence de nombreux grattoirs nécessaires à ce traitement.

Figure 15 - Plan de répartition du mobilier.

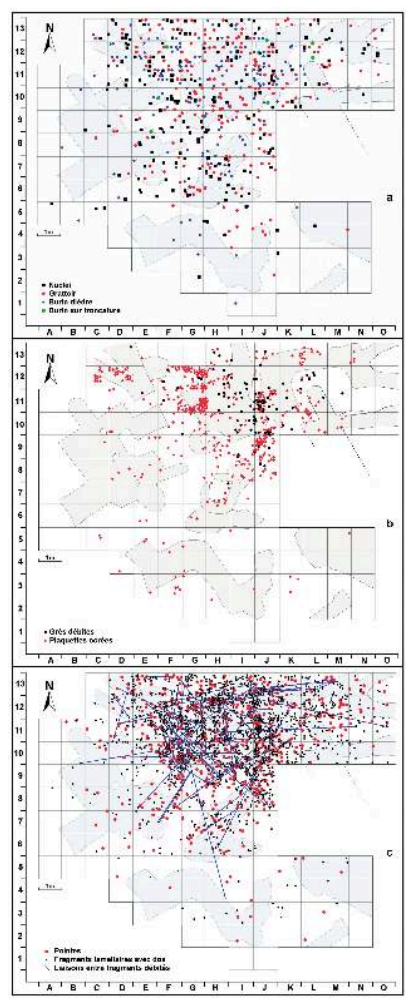

15 a : Nuclei, grattoirs, burins dièdres, burins sur troncature. 15 b : Grès débités et plaquettes ocrées. $15 \mathrm{c}$ : Pointes, fragments lamellaires avec dos et liaisons entre fragments débités.

\section{6 - Conclusions sur la nature et la fonction de ce lieu de vie}

Si les résultats des recherches ont permis de mieux appréhender la nature et le rôle de ce campement, il n'en demeure pas moins que de nombreuses interrogations subsistent et resteront sans réponse en raison de la destruction de la fouille.

111 Malgré l'absence de mobilier osseux due à l'acidité du sol, de nombreux indices permettent de penser qu'il s'agissait d'un camp de base centré sur des activités cynégétiques en bordure de la plaine giboyeuse du Narais. En effet, des armatures, très nombreuses et de types variés, destinées à la chasse y ont été fabriquées et entretenues. La présence dans le foyer 1 de deux grès rainurés ajustables l'un à l'autre incite à penser la mise en forme de hampes de flèches conformes à la finesse et la légèreté des armatures retrouvées sur le site. Cela suggère une diversification des modes de chasse en fonction des gibiers chassés.

112 Le choix de l'implantation du site, l'utilisation intensive des foyers, la présence probable de deux trous de poteau ainsi que de deux zones de sable lessivés renforcent le concept d'habitat aménagé. 
113 Les importantes concentrations de déchets de taille et de retouche associées aux espaces de combustion soulignent que la taille du silex a été réalisée essentiellement près des foyers. Ce constat, conjugué à l'absence de répartition catégorielle ainsi qu'au nombre considérable des produits débités (327000 silex taillés) permet, en conséquence, d'envisager une longue occupation du site par un groupe post-Azilien et une diversité des activités qui y ont été menées en rapport avec la chasse.

\section{BIBLIOGRAPHIE}

ALLARD M. 1977 - Rapport de fouilles sur l'habitat nº 1 du Camp d'Auvours à Saint-Mars-LaBrière (Sarthe), SRA Pays de la Loire.

ALLARD M. 1982 - Un habitat de chasseurs au Camp d'Auvours, in J. COMBIER, Les habitats du Paléolithique supérieur, Actes du colloque international, Roanne-Villerest, 1982, Lyon, Direction des Antiquités Préhistoriques Rhône-Alpes, p. 77-79.

ALLARD M., GUYOT R. 1972 - Etude préliminaire d'une industrie magdalénienne au Camp d'Auvours, commune de Saint-Mars-la-Brière (Sarthe), Bulletin de la Société préhistorique française, 69, p. 347-355.

AUBERT G. et BOULAINE J. 1967 - La pédologie, Paris, Presses Universitaires de France, (Que saisje ?), $128 \mathrm{p}$.

BAISE D. 2004 - Petit lexique de pédologie, Paris, Institut National de la Recherche Agronomique, $271 \mathrm{p}$.

BARTON R.-N.-E. 1986 - Experiments with Long Blades from Spoughton, near Ipswich, Suffolk, In : D.A. Roe dir., Studies in the Hupper Paeolithic of Britain and Northwest Europe, Oxford, Archaeopress (BAR International Series, 296), p. 129-141.

BORDES F. 1952 - Sur l'usage probable de la peinture corporelle dans certaines tribus moustériennes, Bulletin de la Société préhistorique française, 49, p. 169-170.

DUCHAFFOUR P. 2001 - Introduction à la science du sol, abrégé de pédologie, Paris, Dunod, 6e édition, $331 \mathrm{p}$.

GLORY A. 1961 - Le brûloir de Lascaux, Gallia Préhistoire, 4, 4, p. 174-183.

LEROI-GOURHAN A. 1955 - Les hommes de la préhistoire, les chasseurs, Paris, Bourrelier Coll (La joie de connaître), $128 \mathrm{p}$.

LEROI-GOURHAN A. 1976 - Cours du Collège de France 1976, Paris

L'HELGOUACH J. 1971 - Sarthe, Saint-Mars-la-Brière, Gallia Préhistoire,14, 2, p. 371.

L'HELGOUACH J. 1973 - Sarthe, Saint-Mars-la-Brière, Gallia Préhistoire,16, 2, p. 436-437.

L'HELGOUACH J. 1975 - Sarthe, Saint-Mars-la-Brière, Gallia Préhistoire,18, 2, p. 555-558.

L'HELGOUACH J. 1977 - Sarthe, Saint-Mars-la-Brière, Gallia Prehistoire, 20, 2, p. 442-444.

L'HELGOUACH J. 1979 - Sarthe, Saint-Mars-la-Brière, Gallia Prehistoire, 22, 2, p. 577-580. 
MARY G. 1964 - Le Périglaciaire des environs du Mans (Sarthe, France), Reprinted from BIULETYN PERYGLACJALNY, nr 13, Łódź, 98 p.

NAUDINOT N. 2008 - Les armatures lithiques tardiglaciaires dans l'Ouest de la France (Région Bretagne et Pays de la Loire) : proposition d'organisation chrono-culturelle et chaîne opératoire de fabrication. In : J.-M. Pétillon, M.-H. Dias-Meirinho, P. Cattelain, M. Honegger, C. Normand, N. Valdeyron (coord.), Recherches sur les armatures de projectiles du paléolithique supérieur au Néolithique (actes du colloque C83, XVe congrès de l'UISPP, Lisbonne, 4-9 septembre 2006), Palethnologie, 1, p. 250-277.

OBERT D. 1988 - Bouloire, carte géologique de la France à $1 / 50$ 000, Orléans, Bureau de Recherches Géologiques et Minières, carte à 1/50 000 + livret explicatif, 32 p. + guide de lecture, $21 \mathrm{p}$.

POMIES M.-P. 1997 - Pigments rouges préhistoriques : Gœthite chauffée ou hématite nanocristalline naturelle, Thèse de doctorat, Paris VI, 248 p.

ROZOY J.-G. 1978 - Les derniers chasseurs. L'Epipaléolithique en France et en Belgique. Essai de synthèse, Bulletin de la Société archéologique champenoise, 3 vol.

TRIAT J.-M. 2011 - Les ocres, Paris, Centre National de la Recherche Scientifique, 198 p.

VALENTIN B. 1995 - Les groupes humains et leurs traditions au Tardiglaciaire dans le Bassin parisien. Apports de la technologie lithique comparée, Thèse de doctorat, Paris 1, Paris, 834 p., 200 fig.,117 pl.

\section{RÉSUMÉS}

Découverte en 1969, la station préhistorique du Camp militaire d'Auvours a révélé, en surface, la présence de cinq sites. La fouille de $141 \mathrm{~m}^{2}$ réalisée sur le site 1 a livré les restes d'un campement épipaléolithique installé sur un pavement de blocs gréseux d'origine périglaciaire. Ces restes ont révélé des traces d'organisation comportant des zones de combustion, des zones ocrées ainsi que des accumulations de blocs résultant de remaniements d'un pavement naturel.

Le mobilier exclusivement lithique composé d'environ 327000 silex débités dont 4200 retouchés était essentiellement contenu dans la moitié nord du site laissant présager d'une prolongation de celui-ci vers le nord. Les principales nappes de mobilier lithique ont toutes témoigné du débitage du silex ainsi que de la fabrication et de l'entretien d'outils à proximité des foyers. Les armatures surtout représentées par des pointes ( $p$. des Blanchères et $p$. à troncature très oblique) semblent aussi comporter des lamelles à apex naturel décrites ici comme «armatures d'Auvours à apex non aménagé ». Les stigmates observés sur leurs fractures pourraient témoigner de l'usage de l'arc.

La zone d'installation préhistorique a révélé de multiples activités: aménagement de foyers, débitage du silex, préparation de l'ocre, grattage et tannage des peaux. Ce site, témoin pour l'essentiel d'activités cynégétiques, serait donc un important campement de chasseurs. Sa parfaite homogénéité correspond plutôt à une longue et unique occupation post-Azilienne.

Discovered in 1969, the pre-historical site of the military camp of the plateau of Auvours revealed, near the ground surface, the presence of five sites. The $141 \mathrm{~m} 2$ searched area on site 1 show the remains of an epipalaeolithic camp installed on a pavement of sandstone blocks of periglacial origin. This remains revealed traces of organization including combustion zones and linear accumulations of blocks resulting of re-arrangement of the natural pavement. It seems that part of theses accumulations was used to consolidate the base of the walls made up of 
perishable materials. When the search was stopped in 1979, a kind of quadrangular shaped enclosure containing three combustion areas was partially excavated. A supplementary combustion zone, kept in reserve for later survey seemed to lie on the external border of this enclosure in a sector having a large proportion of ochre.

The furniture made up exclusively of stone material, having approximately 327000 cut witnesses of which 4200 were altered, was mainly inside the enclosure and also outside in the sector with ochre. All the main layers of stone products are witnesses of a flint cutting activity as well as production and maintenance of tools located near the hearths.

The armatures are mostly represented by points (points of the Blanchères and points with a very slanted truncation) and seem to have thin strips with natural apex described here as "armatures of Auvours". The stigmatas observed on their fractures might be testimonies of the use of bow.

The configuration of the quadrangular area suggest for its protection a single covering comparable with the ones of Thuareg tent. Its North part centralizing miscellaneous material cutting and ochre processing activities constituted a very busy craftsman space which reveals occupations probably associated to hunting. This site, witness mostly of hunting activities, could therefore have been an important camp site for hunters. Its perfect homogeneity rather corresponds to a long and single occupation. Finally, having no reliable dating, it seems preferable in principle to put forward the hypothesis that this vast camp site, with an opening on each side, did not coincide with the cold phase of Dryass III.

\section{INDEX}

Keywords : Epipalaeolithic, camp site of hunters, hunting activitie, combustion area, accumulation of blocs, natural pavement, flat ferruginous granule, point of the Blanchères, armature of Auvours

Mots-clés : Post-Azilien, campement de chasseurs, activité cynégétique, foyer, accumulation de blocs, pavement naturel, plaquette ocrée, pointe des Blanchères, armature d'Auvours à apex aménagé

\section{AUTEUR}

\section{MICHEL ALLARD}

21, rue de Giroussens, FR-31500 Toulouse

michelallard@free.fr 